\title{
NEW APPROACH TO ASYMPTOTIC STABILITY: TIME-VARYING NONLINEAR SYSTEMS
}

\author{
L.T. GRUJIĆ \\ Ecole Nationale d'Ingenieurs de Belfort \\ Espace Bartholdi, Belfort Technopole, B.P. 525 \\ 90016 Belfort Cedex - FRANCE
}

(Received February 24, 1994 and in revised form June 30, 1995)

\begin{abstract}
The results of the paper concern a broad family of time-varying nonlinear systems with differentiable motions. The solutions are established in a form of the necessary and sufficient conditions for: 1) uniform asymptotic stability of the zero state, 2) for an exact single construction of a system Lyapunov function and 3) for an accurate single determination of the (uniform) asymptotic stability domain. They permit arbitrary selection of a function $p(\cdot)$ from a defined functional family to determine a Lyapunov function $\mathbf{v}(\bullet),[v(\bullet)]$, by solving $v^{\prime}(\bullet)=-p(\bullet)$ (or equivalently, $v^{\prime}(\bullet)=-p(\bullet)[1-v(\bullet)\}$, respectively. Illustrative examples are worked out.
\end{abstract}

KEY WORDS AND PHRASES: Nonlinear Dynamical Ssytems, Lyapunov and Asymptotic Stability. 1991 AMS SUBJECT CLASSIFICATION CODES: 34A34, 34C35, 34D20, 93D05, 93D20.

\section{INTRODUCTION}

The well known fundamental advantageous feature of the Lyapunov method consists in the use of both a positive definite function and its total derivative along system motions without knowing the motions themselves in order to investigate qualitative properties of the system behavior, among which there are various asymptotic stability properties.

Theorems established for time-varying nonlinear systems have been expressed in terms of existence of a Lyapunov function $\mathrm{v}(\bullet)[v(\bullet)]$ without clarifying how to determine it, that is without clarifying how to choose $p(\cdot)$ in $v^{\prime}(\cdot)=-p(\cdot)$ \{or equivalently, in $v^{\prime}=-p(\cdot)[1-v(\cdot)\}$, and what are, with respect to a selected $p(\bullet)$, the necessary and sufficient conditions for a solution $\mathbf{v}(\bullet)[v(\bullet)]$, respectively, to guarantee uniform asymptotic stability of $x=0$ and/or to determine accurately its domain of uniform asymptotic stability. Such a crucial incompleteness of the existing Lyapunov stability theory has been an inherent obstacle to broader and more effective applications of the theory than have been realized. It was overcome in [2]-[12] for different classes of time-invariant systems by proposing three distinct approaches. Their common feature is in defining a family of functions $p(\bullet)$ used to generate a function $\mathrm{v}(\bullet)[o r, v(\bullet)]$ and in specifying the necessary and sufficient properties of $\mathrm{v}(\bullet)$ [or, $v(\bullet)$ ] to guarantee asymptotic stability of the zero state and/or to ensure that a set $N$ is the domain of its asymptotic stability. This paper is aimed to establish analogous solutions for a broad family of time-varying nonlinear systems.

\section{NOTATION}

Capital italic Roman letters are used for sets, lower case block Roman characters for vectors, Greek letters and lower case italic letters denote scalars except for the empty set $\emptyset$ and subscripts. The boundary, interior and closure of a set $A$ are designated by $\partial A, \operatorname{In} A$ and $\mathrm{Cl} A$, respectively, where $A$ is a time-invariant set. If $A(\bullet): R \rightarrow 2^{R^{n}}$ is a set-valued function then its instantaneous set value $A(t)$ at arbitrary time $t \in R$ will be called a time-varying set $A(t)$. Let $\|\bullet\|: R^{n} \rightarrow R_{+}$be the Euclidean 
norm on $R^{n}$, where $R_{+}=\left[0, \infty\left[=\{x: x \in R, 0 \leq x<\infty\} . \quad B_{\alpha}\right.\right.$ will be used for the open hyperball with radius $\alpha$ centered at the origin, $B_{\alpha}=\left\{\mathbf{x}: \mathbf{x} \in R^{n},\|\mathbf{x}\|<\alpha\right\}$. An initial time $t_{0} \in R_{1}$ and determines $R_{0}=\left[t_{0}, \infty\left[\right.\right.$, where $\left.R_{i}=\right] \sigma,+\infty[, \sigma \in[-\infty, \infty[$.

Let $\left.\zeta \in R^{+}=\right] 0, \infty\left[\right.$ and $p(\cdot): R_{\imath} \times R^{n} \rightarrow R$. Then $P_{\zeta}(t)$ is the largest open connected neighborhood of $\mathrm{x}=0$ at time $t \in R_{i}$ such that $p(t, \mathrm{x})<\zeta$ for every $\mathrm{x} \in P_{\zeta}(t)$.

Let $\rho(\bullet): R^{n} \times 2^{R^{n}} \rightarrow R_{+}$be a distance function defined by

$$
\rho(\mathrm{x}, A)=\inf [\|\mathrm{x}-\mathrm{y}\|: \mathrm{y} \in A] .
$$

Let now $\mathrm{d}(\bullet): 2^{R^{n}} \times 2^{R^{n}} \rightarrow R_{+}$be a distance function introduced by:

$$
\mathrm{d}(A, B)=\max \{[\sup \rho(\mathrm{x}, B): \mathrm{x} \in A],[\sup \rho(\mathrm{y}, A): \mathrm{y} \in B]\} .
$$

A non-empty set-valued function $S(\bullet): R \rightarrow 2^{R^{n}}$ is continuous at $\tau \in R$ if and only if for every $\epsilon \in R^{+}$there is $\delta \in R^{+}, \delta=\delta(\tau, \epsilon)$, such that $|t-\tau|<\delta$ implies $\mathrm{d}[S(t), S(\tau)]<\epsilon$. It is continuous on $R_{0}$ (i.e. in $t \in R_{0}$ ) if and only if it is continuous at every $t \in R_{0}$.

Let $\mathbf{x}\left(\bullet ; t_{0}, \mathrm{x}_{0}\right)$ be a motion (solution) of a system through $\mathrm{x}_{0} \in R^{n}$ at $t_{0} \in R_{i}$, and $\mathbf{x}\left(t ; t_{0}, \mathrm{x}_{0}\right)$ be its vector value $\mathrm{x}(t)$ at time $t \in R_{i}, \mathbf{x}(t) \equiv \mathbf{x}\left(t ; t_{0}, \mathrm{x}_{0}\right)$.

If a function $\mathrm{x}(\cdot)$ is differentiable then its total time derivative $\mathrm{dx}(\cdot) / \mathrm{d} t$ will be also denoted by $\mathrm{x}^{\prime}(\bullet)$. If $v: R_{i} \times R^{n} \mapsto R$ is differentiable then its total time derivative along $\mathrm{x}(\cdot)$ is its Eulerian derivative $v[\bullet, x(\cdot)]$,

$$
v[t, \mathbf{x}(t)]=\frac{\partial v[t, \mathbf{x}(t)]}{\partial t}+\{\operatorname{grad} v[t, \mathbf{x}(t)]\}^{\mathrm{T}} \mathbf{x}^{\prime}(t) .
$$

The notation used for stability domains is explained in Definitions 1-3 (Section 4). Their importance was explained by LaSalle and Lefschetz [17].

\section{SYSTEM DESCRIPTION}

Nonlinear time-varying systems treated herein are described by (3.1),

$$
\frac{\mathrm{dx}(t)}{\mathrm{d} t}=\mathrm{f}[t, \mathrm{x}(t)], \quad \mathrm{x}(\cdot): R \rightarrow R^{n}, \mathrm{f}(\cdot): R \times R^{n} \rightarrow R^{n},
$$

and by one of the following features:

\section{Weak Smoothness Property}

(i) There is an open continuous neighborhood $S(t)$ of $\mathrm{x}=0$ for every $t \in R_{i}, S(t) \subseteq R^{n}$, such that $S=\cap\left[S(t): t \in R_{i}\right]=S\left(R_{2}\right)$ is also an open neighborhood of $\mathrm{x}=0$ and for every $\left(t_{0}, \mathrm{x}_{0}\right) \in R_{i} \times S\left(t_{0}\right)$ :

a) the system (3.1) has a unique solution $x\left(\bullet ; t_{0}, x_{0}\right)$ through $x_{0}$ at $t_{0}$ on a largest interval $I_{0}$, $I_{0}=I_{0}\left(t_{0}, x_{0}\right), R_{i} \supseteq I_{0}, I_{0} \neq \emptyset$, and

b) $x\left(\bullet ; t_{0}, x_{0}\right)$ is defined, continuous and differentiable in $\left(t, t_{0}, x_{0}\right) \in I_{0} \times R_{i} \times S\left(t_{0}\right)$.

(ii) For every $\left(t_{0}, \mathrm{x}_{0}\right) \in R_{i} \times\left[R^{n}-\partial S\left(t_{0}\right)\right]$ every motion $\mathbf{x}\left(\cdot ; t_{0}, \mathrm{x}_{0}\right)$ of the system $(3.1)$ is continuous in $t \in I_{0}$.

\section{Strong Smoothness Property}

(i) The system (3.1) obeys the Weak Smoothness Property.

(ii) If the boundary $\partial S(t)$ of $S(t)$ is non-empty at any $\tau \in R_{i}$ then every motion of the system (3.1) passing through $\mathrm{x}_{0} \in \partial S\left(t_{0}\right)$ at $t_{0}=\tau$ obeys $\inf \left[\left\|\mathbf{x}\left(t ; t_{0}, \mathbf{x}_{0}\right)\right\|: t \in I_{0}\right]>0$ for every $\left(t_{0}, \mathrm{x}_{0}\right) \in R_{i} \times \partial S\left(t_{0}\right)$.

\section{ASYMPTOTIC STABILITY DOMAINS}

This section is aimed to clarify the notions of the asymptotic stability domains in the framework of time-varying systems. 
The notion of the attraction domain has been mainly used in the following sense (Hahn [15], Zubov [20]):

\section{Definition 1.}

The state $x=0$ of the system (3.1) has:

(a) the domain of attraction at $t_{0}$ denoted by $D_{a}\left(t_{0}\right), D_{a}\left(t_{0}\right) \subseteq R^{n}$, if and only if both 1) and 2) hold,

1) for $t_{0} \in R$ and every $\zeta \in R^{+}$there exists $\tau=\tau\left(t_{0}, \mathrm{x}_{0}, \zeta\right) \in R_{+}$such that $\left\|\mathbf{x}\left(t ; t_{0}, \mathrm{x}_{0}\right)\right\|<\zeta$ for all $t \in] t_{0}+\tau, \infty\left[\right.$ is valid provided only that $x_{0} \in D_{a}\left(t_{0}\right)$,

2) the set $D_{a}\left(t_{0}\right)$ is a neighborhood of $\mathrm{x}=0$.

(b) the domain $D_{a}\left(R_{i}\right)$ of uniform attraction on $R_{i}, D_{a}\left(R_{i}\right) \subseteq R^{n}$, if and only if 1)-4) hold,

1) it has the domain $D_{a}\left(t_{0}\right)$ of attraction at every $t_{0} \in R_{i}$,

2) $\cap\left[D_{a}\left(t_{0}\right): t_{0} \in R_{i}\right]$ is a neighborhood of $x=0$,

3) $D_{a}\left(R_{i}\right)=\cap\left[D_{a}\left(t_{0}\right): t_{0} \in R_{i}\right]$,

4) the minimal $\tau\left(t_{0}, \mathrm{x}_{0}, \zeta\right)$ obeying 1$)$ of (a) and denoted by $\tau_{\mathrm{m}}\left(t_{0}, \mathrm{x}_{0}, \zeta\right)$ satisfies

$$
\sup \left[\tau_{\mathrm{m}}\left(t_{0}, \mathrm{x}_{0}, \zeta\right): t_{0} \in R_{i}\right]<+\infty \text {, for every }\left(\mathrm{x}_{0}, \zeta\right) \in D_{a}\left(R_{l}\right) \times R^{+} .
$$

The expression "on $R_{i}$ " is to be omitted if and only if $R_{i}=R$. Then and only then $D_{a}\left(R_{i}\right)$ will be denoted by $D_{a}, D_{a}=D_{a}(R)$.

The stability domain and the asymptotic stability domain in the Lyapunov sense were introduced in [1], and further broadened and used in [2]-[14] as follows:

\section{Definition 2.}

The state $x=0$ of the system (3.1) has:

(a) the domain of stability at $t_{0}$ denoted by $D_{s}\left(t_{0}\right), D_{s}\left(t_{0}\right) \subseteq R^{n}$, if and only if 1)-3) hold,

1) for every $\epsilon \in R^{+}$the motion $\mathbf{x}\left(\cdot ; t_{0}, \mathbf{x}_{0}\right)$ satisfies $\left\|\mathbf{x}\left(t ; t_{0}, \mathbf{x}_{0}\right)\right\|<\epsilon$ for all $t \in R_{0}$ provided only that $\mathrm{x}_{0} \in D_{s}\left(t_{0}, \epsilon\right)$,

2) the set $D_{s}\left(t_{0}, \epsilon\right)$ is a neighborhood of $\mathrm{x}=0$ for every $\epsilon \in R^{+}$,

3) the set $D_{s}\left(t_{0}\right)$ is the union of all the sets $D_{s}\left(t_{0}, \epsilon\right)$ over $\epsilon \in R^{+}$:

$$
D_{s}\left(t_{0}\right)=\cup\left[D_{s}\left(t_{0}, \epsilon\right): \epsilon \in R^{+}\right] \text {. }
$$

(b) the domain $D_{s}\left(R_{i}\right)$ of uniform stability on $R_{i}$ if and only if 1)-3) hold,

1) it has the domain $D_{s}\left(t_{0}\right)$ of stability at every $t_{0} \in R_{h}$,

2) $\cap\left[D_{s}\left(t_{0}\right): t_{0} \in R_{i}\right]$ is a neighborhood of $x=0$,

3) $D_{s}\left(R_{i}\right)=\cap\left[D_{s}\left(t_{0}\right): t_{0} \in R_{i}\right]$.

The expression "on $R_{i}$ " is to be omitted if and only if $R_{2}=R$. Then and only then $D_{s}\left(R_{1}\right)$ will be denoted by $D_{s}, D_{s}=D_{s}(R)$.

\section{Definition 3.}

The state $x=0$ of the system (3.1) has:

(a) the domain of asymptotic stability at $t_{0}$ denoted by $D\left(t_{0}\right), D\left(t_{0}\right) \subseteq R^{n}$, if and only if it has both $D_{a}\left(t_{0}\right)$ and $D_{s}\left(t_{0}\right)$, and $D\left(t_{0}\right)$ is their intersection:

$$
D\left(t_{0}\right)=D_{a}\left(t_{0}\right) \cap D_{s}\left(t_{0}\right) .
$$

(b) the domain $D\left(R_{i}\right)$ of uniform asymptotic stability on $R_{i}$ if and only if it has both $D_{a}\left(R_{i}\right)$ and $D_{s}\left(R_{i}\right)$, and $D\left(R_{l}\right)$ is their intersection:

$$
D\left(R_{i}\right)=D_{a}\left(R_{i}\right) \cap D_{s}\left(R_{i}\right) .
$$

The expression "on $R_{i}$ " is to be omitted if and only if $R_{i}=R$. Then and only then $D\left(R_{1}\right)$ will be denoted by $D, D=D(R)$. 
Qualitative features of the stability domains of $x=0$ of the system (3.1) are discovered and proved in Appendix 1. They are important for proofs of the main results of the paper (Section 6).

\section{FAMIILIES OF FUNCTIONS $p(\bullet)$ AND LYAPUNOV FUNCTIONS}

Families $P(\bullet)$ and $P^{1}(\bullet)$ of functions $p(\bullet)$ were used in [10], [11] to generate Lyapunov functions $v(\bullet)$ obtained as solutions of $v^{\prime}(\bullet)=-p(\bullet)$ [or, to determine Lyapunov functions $v(\bullet)$ as solutions of $\left.v^{\prime}(\bullet)=-[1-v(\cdot)] p(\bullet)\right]$ in the framework of time invariant systems. In the setting of this paper they will be replaced by families $L^{1}(\bullet)$ and $E^{1}(\bullet)$ of functions $p(\bullet)$.

\section{Definition 4.}

A function $p(\bullet): R_{2} \times R^{n} \rightarrow R$ belongs to family $L^{1}\left(R_{i}, S ; \mathrm{f}\right)$ if and only if:

1) $p(\bullet)$ is differentiable on $R_{i} \times S: p(t, \mathrm{x}) \in C^{(1)}\left(R_{i} \times S\right)$,

2) the equations (5.1) with (5.1a) taken along motions of the system (3.1),

$$
\begin{gathered}
v^{\prime}(t, \mathbf{x})=-p(t, \mathbf{x}), \\
v(t, 0)=0, \quad \forall t \in R_{i},
\end{gathered}
$$

have a solution $v(\bullet)$ that is continuous and differentiable in $(t, \mathrm{x}) \in R_{i} \times \mathrm{ClB} B_{\mu}$ for some (anyhow small) $\mu \in R^{+}, \mu=\mu(\mathrm{f}, p)$, and which obeys (5.2) for some $w_{\mu}(\mathrm{x}) \in C\left(\mathrm{ClB} B_{\mu}\right)$,

$$
v(t, \mathbf{x}) \leq w_{\mu}(\mathbf{x}), \forall(t, \mathbf{x}) \in R_{i} \times \mathrm{ClB}_{\mu},
$$

and

3) for any $\rho \in R^{+}$fulfilling $S(t) \supset \mathrm{ClP} P_{\rho}(t)$ for all $t \in R_{i}$ it holds:

$$
\min \left\{p(t, \mathbf{x}):(t, \mathbf{x}) \in R_{i} \mathbf{x}\left[S(t)-P_{\rho}(t)\right]\right\}=\alpha, \alpha=\alpha(\rho ; p) \in R^{+} .
$$

\section{Definition 5.}

A function $p(\cdot): R_{\imath} \times R^{n} \rightarrow R$ belongs to family $E^{1}\left(R_{i}, S ; f\right)$ if and only if:

1) $p(\bullet)$ is differentiable on $R_{i} \times S: p(t, x) \in C^{(1)}\left(R_{i} \times S\right)$,

2) the equations (5.3) with (5.3a) taken along motions of the system (3.1),

$$
\begin{gathered}
v^{\prime}(t, \mathbf{x})=-[1-v(t, \mathbf{x})] p(t, \mathbf{x}), \\
v(t, 0)=0, \quad \forall t \in R_{i},
\end{gathered}
$$

have a solution $v$ that is differentiable in $(t, \mathrm{x}) \in R_{i} \times \mathrm{ClB}_{\mu}$ for some $\mu \in R^{+}, \mu=\mu(\mathrm{f}, p)$, and which obeys (5.4) for some $w_{\mu}(x) \in C\left(C l B_{\mu}\right)$,

$$
v(t, \mathbf{x}) \leq w_{\mu}(\mathbf{x}), \quad \forall(t, \mathbf{x}) \in R_{i} \times \mathrm{ClB}_{\mu}
$$

and

3) for any $\rho \in R^{+}$satisfying $S(t) \supset \mathrm{Cl} P_{\rho}(t)$ for all $t \in R_{i}$ it holds:

$$
\min \left\{p(t, \mathbf{x}):(t, \mathbf{x}) \in R_{i} \mathbf{x}\left[S(t)-P_{\rho}(t)\right]\right\}=\alpha, \alpha=\alpha(\rho ; p) \in R^{+} .
$$

Notice that $p(\bullet) \in L^{1}\left(R_{i}, S, \mathrm{f}\right)$ if and only if $p(\bullet) \in E^{1}\left(R_{i}, S, \mathrm{f}\right)$, which is easy to verify. If $p(\bullet) \in L^{1}\left(R_{i}, S ; \mathrm{f}\right)$, hence $p(\bullet) \in E^{1}\left(R_{\imath}, S, \mathrm{f}\right)$, then solutions $v(\bullet)$ and $v(\bullet)$ to $(5.1)$ and $(5.3)$ are interrelated by (5.5),

$$
v(t, \mathbf{x})=1-\exp [-v(t, \mathbf{x})],
$$

which was pointed out by Vanelli and Vidyasagar [19]. Besides, $v(t, \mathbf{x})=0$ if and only if $\nu(t, \mathbf{x})=0$, and $v(t, \mathbf{x}) \rightarrow 1$ if and only if $v(t, \mathbf{x}) \rightarrow \infty$. 
There is not any stability condition imposed on the system and no definiteness requirement is imposed on $p(\bullet), v(\bullet)$ and $v(\bullet)$ in Definition 4 and Definition 5 Therefore, $L^{1}\left(R_{h}, S, \mathrm{f}\right)$ and $E^{1}\left(R_{h}, S, \mathrm{f}\right)$ are not dependent in general on a stability property of the system.

\section{NOVEL SOLUTIONS TO THE CLASSICAL STABILITY PROBLEMS}

For the sake of clearness we emphasize that the notions of a positive definite function and of a decrescent function will be used in the usual sense (c.f Hahn [15], Zubov [20]), that is that a function $v(\cdot): R_{2} \times R^{n} \rightarrow R$

(a) is positive definite on $R_{2} \times A(t)$ if and only if $A=\cap\left[A(t): t \in R_{l}\right]$ is an open connected neighborhood of $\mathrm{x}=0$ such that there exists $w_{1}(\bullet): R^{n} \rightarrow R$ obeying the following

1) $v(t, \mathbf{x})$ and $w_{1}(\mathbf{x})$ are uniquely determined by $(t, \mathbf{x}) \in R_{2} \times A(t)$ and continuous on $R_{2} \times A(t)$, $v(t, \mathrm{x})$ is also differentiable in $(t, \mathrm{x}) \in R_{\imath} \times A(t)$, that is $v(t, \mathrm{x}) \in C^{(1)}\left[R_{\imath} \times A(t)\right]$ and $w_{1}(\mathbf{x}) \in C\left\{\cup\left[A(t): t \in R_{1}\right]\right\}$,

2) $v(t, 0)=0$ for all $t \in R_{h}, w_{1}(0)=0$,

and

3) $v(t, \mathbf{x}) \geq w_{1}(\mathbf{x})$ for all $(t, \mathbf{x}) \in R_{\imath} \times A(t)$.

(b) is 'decrescent on $R_{2} \times A(t)$ if and only if $A=\cap\left[A(t): t \in R_{1}\right]=A\left(R_{1}\right)$ is open connected neighborhood of $\mathrm{x}=0$ such that there exists $w_{2}(\bullet): R^{n} \rightarrow R$ obeying what follows

1) $v(t, \mathbf{x})$ and $w_{2}(\mathbf{x})$ are continuous on $R_{2} \times A$, that is $v(t, \mathbf{x}) \in C\left(R_{2} \times A\right)$ and $w_{2}(\mathbf{x}) \in C(A)$, and

2) $v(t, \mathbf{x}) \leq w_{2}(\mathbf{x})$ for all $(t, \mathbf{x}) \in R_{\imath} \times A$.

The expression " $R_{i}$ " is to be omitted if and only if $R_{h}=R$, and the expression "х $A(t)$ " is to be omitted if and only if $A(t)$ is some (anyhow small) open connected neighborhood of $\mathrm{x}=0$ for all $t \in R_{\mathrm{h}}$.

Solutions to the problems will depend on the smoothness properties of the system (3.1) as well as whether a function $p(\bullet)$ generating a system Lyapunov function is selected from $L^{1}\left(R_{\imath}, S, \mathrm{f}_{r}\right)$ or from $E^{1}\left(R_{\imath}, S, \mathrm{f}\right)$

THEOREM 1. For the state $\mathrm{x}=0$ of the system (3.1) with the Strong Smoothness Property to have the domain $D\left(R_{2}\right)$ of uniform asymptotic stability on $R_{2}$, for a set $N\left(t_{0}\right), N\left(t_{0}\right) \subseteq R^{n}$, to be the domain of its asymptotic stability at $t_{0} \in R_{\imath}: N\left(t_{0}\right) \equiv D\left(t_{0}\right)$, and for a set $N, N \subseteq R^{n}$, to be the domain of its uniform asymptotic stability on $R_{h}, N=D\left(R_{2}\right)$, it is both necessary and sufficient that

1) the set $N\left(t_{0}\right)$ is an open neighborhood of $\mathrm{x}=0$ and $N\left(t_{0}\right) \subseteq S\left(t_{0}\right)$ for every $t_{0} \in R_{\imath}$,

2) the set $N$ is a connected neighborhood of $\mathrm{x}=0$ and $N \subseteq S$,

3) $\mathrm{f}(t, \mathrm{x})=0$ for $(t, \mathrm{x}) \in R_{2} \times N(t)$ if and only if $\mathrm{x}=0$,

and

4) for any differentiable decrescent positive definite function $p(\cdot)$ on $R_{\imath} \times S(t)$ obeying:

(a) $p(\bullet) \in L^{1}\left(R_{\imath}, S ; \mathrm{f}\right)$ the equations (5.1) have the unique solution function $v(\cdot)$ with the following properties.

(i) $v(\bullet)$ is a decrescent positive definite function on $R_{\imath} \times N(t)$,

(ii) if the boundary $\partial N(t)$ of $N(t)$ is nonempty then $\mathrm{x} \rightarrow \partial N(t), \mathrm{x} \in N(t)$, implies $v(t, \mathbf{x}) \rightarrow \infty$ for every $t \in R_{\imath}$, 
and

(iii) $N=\cap\left[N(t): t \in R_{l}\right]=N\left(R_{2}\right)$,

or obeying:

(b) $p(\bullet) \in E^{1}\left(R_{\imath}, S ; \mathrm{f}\right)$ the equations (5.3) have the unique solution function $v(\bullet)$ with the following properties:

(i) $v(\bullet)$ is a decrescent positive definite function on $R_{\imath} \times N(t)$,

(ii) if the boundary $\partial N(t)$ of $N(t)$ is nonempty then $\mathrm{x} \rightarrow \partial N(t), \mathrm{x} \in N(t)$, implies $v(t, \mathbf{x}) \rightarrow 1$ for every $t \in R_{\imath}$,

and

(iii) $N=\cap\left[N(t): t \in R_{l}\right]=N\left(R_{l}\right)$.

PROOF. Necessity. Let $\mathrm{x}=0$ of the system (3.1) possessing the Strong Smoothness Property have the uniform asymptotic stability domain $D\left(R_{l}\right)$ on $R_{\imath}$ Hence, it has also the asymptotic stability domain $D\left(t_{0}\right)$ at every $t_{0} \in R_{h}$ (Definition 1). Definitions 1 and 3 show that it has also the uniform attraction domain $D_{a}\left(R_{1}\right)$ and the attraction domain $D_{a}\left(t_{0}\right)$ at every $t_{0} \in R_{l}$. Obviously, $D_{a}\left(t_{0}\right) \supseteq D\left(t_{0}\right)$ for all $t_{0} \in R_{\imath}$ and $D_{a}\left(R_{\imath}\right) \supseteq D\left(R_{\imath}\right)$. Besides, $D_{a}\left(t_{0}\right)$ is a neighborhood of $\mathrm{x}=0$ at every $t_{0} \in R_{l}$ and $D_{a}\left(R_{1}\right)$ is also a neighborhood of $\mathrm{x}=0$ (Definition 1) The set $S\left(t_{0}\right)$ is a neighborhood of $\mathrm{x}=0$ at every $t_{0} \in R_{1}$ and $S$ is also a neighborhood of $\mathrm{x}=0$ (the Weak Smoothness Property). Hence, $D_{a}\left(t_{0}\right) \cap S\left(t_{0}\right) \neq \emptyset$ for all $t_{0} \in R_{l}$ and $D_{a}\left(R_{l}\right) \cap S \neq \emptyset$. Let us prove $S\left(t_{0}\right) \supseteq D_{a}\left(t_{0}\right)$ for every $t_{0} \in R_{l}$. If $\partial S\left(t_{0}\right) \neq \emptyset$ then we will consider separately $\mathrm{u} \in \partial S\left(t_{0}\right)$ and $\mathrm{w} \in\left[R^{n}-\mathrm{ClS}\left(t_{0}\right)\right]$. If $\mathrm{u} \in \partial S\left(t_{0}\right)$ then $\mathrm{u} \notin \partial D_{a}\left(t_{0}\right)$ due to (ii) of the Strong Smoothness Property. Therefore, $D_{a}\left(t_{0}\right) \cap \partial S\left(t_{0}\right)=\emptyset, \forall t_{0} \in R_{\text {r }}$. If $\mathbf{w} \in\left[R^{n}-\mathrm{Cl} S\left(t_{0}\right)\right]$ then for $\mathbf{x}\left(t ; t_{0}, \mathbf{w}\right) \rightarrow 0$ as $t \rightarrow \infty$ it is necessary that there is $t^{*} \in R_{0}$ such that $\mathbf{x}\left(t^{*} ; t_{0}, \mathbf{w}\right) \in \partial S\left(t^{*}\right)$ because $D_{a}(t)$ and $S(t)$ are neighborhoods of $\mathbf{x}=0$ for all $t_{0} \in R_{i}$, both $S(t)$ and $\mathbf{x}\left(t ; t_{0}, w\right)$ are continuous in $t \in R_{4}$ and $S=\cap\left[S(t): t \in R_{l}\right]$ is an open neighborhood of $\mathrm{x}=0$ (the Weak Smoothness Property). However, $\mathbf{x}\left(t^{*} ; t_{0}, \mathbf{w}\right) \in \partial S\left(t^{*}\right)$ implies inf $\left[\left\|\mathbf{x}\left(t ; t_{0}, \mathbf{w}\right)\right\|: t \in R_{0}\right]>0$ because of (ii) of the Strong Smoothness Property. This yields $\mathrm{w} \notin \partial D_{a}\left(t_{0}\right)$ and $\left[R^{n}-\mathrm{ClS}\left(t_{0}\right)\right] \cap D\left(t_{0}\right)=\emptyset, \forall t_{0} \in R_{h}$ Altogether, $D_{a}\left(t_{0}\right) \cap S\left(t_{0}\right) \neq \emptyset, \quad D_{a}\left(t_{0}\right) \cap \partial S\left(t_{0}\right)=\emptyset, \quad D_{a}\left(t_{0}\right) \cap\left[R^{n}-C l S\left(t_{0}\right)\right]=\emptyset, \quad \forall t_{0} \in R_{l}$, which prove $S\left(t_{0}\right) \supseteq D_{a}\left(t_{0}\right), \forall t_{0} \in R_{l}$. Therefore, $S\left(t_{0}\right) \supseteq D\left(t_{0}\right)$ due to $D_{a}\left(t_{0}\right)=D\left(t_{0}\right)$ (Lemma A.2). Let $N\left(t_{0}\right) \equiv D\left(t_{0}\right)$ so that $S\left(t_{0}\right) \supseteq N\left(t_{0}\right)$. Hence, $N\left(t_{0}\right)$ is an open neighborhood of $\mathrm{x}=0$ (Lemma A.1), which proves necessity of the condition 1). Now, $N=D$ and Lemma A.1 prove necessity of the condition 2). From $D_{s}(t) \supseteq D_{a}(t) \equiv D(t) \equiv N(t)$ and Definitions 1-3 it results that $\mathrm{x}=0$ is the unique equilibrium state of $\mathrm{x}=0$ of the system (3 1) in $N(t), \forall t_{0} \in R_{\imath}$, which implies $f(t, \mathbf{x})=0$ for $(t, \mathrm{x}) \in R_{1} \times N(t)$ if and only if $\mathrm{x}=0$ (Proposition 7 in Grujić et al. [12]) This proves necessity of the condition 3). From $N\left(t_{0}\right) \equiv D\left(t_{0}\right)$ it follows that the interval $I_{0}$ of existence of $\mathbf{x}\left(\cdot ; t_{0}, \mathrm{x}_{0}\right)$ satisfies $I_{0} \subseteq R_{0}, \forall\left(t_{0}, \mathrm{x}_{0}\right) \in R_{1} \times N\left(t_{0}\right)$ due to Definitions 1-3. Let $p(\bullet) \in L^{1}\left(R_{2}, S, \mathrm{f}\right)$ be an arbitrarily selected positive definite decrescent function on $R_{L} \times S(t)$ Hence, there is $\mu>0$ such that there exists a solution function $v(\cdot)$ to the equations (5.1), which is continuous in $(t, \mathrm{x}) \in R_{\imath} \times \mathrm{ClB}_{\mu}$ and satisfies (5.2). Therefore,

$$
\begin{gathered}
|v(t, \mathbf{x})|<\infty, \quad \forall(t, \mathbf{x}) \in R_{\imath} \times \mathrm{ClB}_{\mu}, \\
|\partial v(t, \mathbf{x}) / \partial t|<\infty, \quad \forall(t, \mathbf{x}) \in R_{\imath} \times \mathrm{ClB}_{\mu},
\end{gathered}
$$

and 


$$
\|\operatorname{grad} v(t, \mathbf{x})\|<\infty, \quad \forall(t, \mathbf{x}) \in R_{i} \times \mathrm{ClB}_{\mu} .
$$

Let $\beta \in] 1, \infty\left[\right.$ and $\zeta \in R^{+}$be such that

$$
\mathrm{ClB}_{\beta} \cap \mathrm{ClB} B_{\mu} \cap S(t) \supset P_{\zeta}(t), \quad \forall t \in R_{\imath} .
$$

Existence of such $\zeta$ is guaranteed by positive definiteness of $p(\bullet)$ on $R_{l} \times S(t)$ and the fact that $\cap\left[S(t): t \in R_{i}\right]$ is a neighborhood of $\mathrm{x}=0$. Let $t_{0} \in R_{i}$ be arbitrary and $\tau \in R_{+}$, $\tau=\tau\left(t_{0}, \mathrm{x}_{0} ; \mathrm{f}, p, \zeta\right)$, be such that for any $\mathrm{x}_{0} \in N\left(t_{0}\right)$ the condition (6.3) holds,

$$
\mathbf{x}\left(t ; t_{0}, \mathbf{x}_{0}\right) \in \operatorname{ClP}_{\zeta}(t), \quad \forall t \in\left[t_{0}+\tau, \infty[\right.
$$

Such $\tau$ exists due to Definitions 1 and $3, \mathrm{x}_{0} \in N\left(t_{0}\right)$ and $D_{a}\left(t_{0}\right) \equiv D\left(t_{0}\right) \equiv N\left(t_{0}\right)$. Notice that $\mathrm{x}_{0} \in N\left(t_{0}\right)$ yields also

$$
\mathbf{r}\left(\infty ; t_{0}, \mathbf{x}_{0}\right)=0
$$

Let (5.1a) be integrated from $t \in R_{0}$ to $\infty$,

$$
v\left[\infty, \mathbf{x}\left(\infty ; t_{0}, \mathrm{x}_{0}\right)\right]-v\left[t, \mathbf{x}\left(t ; t_{0}, \mathrm{x}_{0}\right)\right]=-\int_{t}^{\infty} p\left[\sigma, \mathbf{x}\left(\sigma ; t_{0}, \mathrm{x}_{0}\right)\right] d \sigma, \quad \forall\left(t, \mathbf{x}_{0}\right) \in R_{0} \times N\left(t_{0}\right),
$$

Now, (5.1b) and (6.4) yield (6.5) in the next form,

$$
v\left[t, \mathbf{x}\left(t ; t_{0}, \mathbf{x}_{0}\right)\right]=\int_{t}^{\tau} p\left[\sigma, \mathbf{x}\left(\sigma ; t_{0}, \mathrm{x}_{0}\right)\right] \mathrm{d} \sigma+\int_{\tau}^{\infty} p\left[\sigma, \mathbf{x}\left(\sigma ; t_{0}, \mathrm{x}_{0}\right)\right] d \sigma, \quad \forall\left(t, \mathbf{x}_{0}\right) \in R_{0} \times N\left(t_{0}\right) .
$$

Invariance of $D_{a}(t)$ with respect to system motions on $R_{i}$ (Lemma A.1), $S(t) \supseteq D(t) \equiv D_{a}(t) \equiv N(t)$, differentiability of the motions $\mathbf{x}\left(t ; t_{0}, \mathrm{x}_{0}\right)$ in $\left(t ; t_{0}, \mathrm{x}_{0}\right) \in I_{0} \times R_{\imath} \times S\left(t_{0}\right)$ [(i-b) of the Weak Smoothness Property], continuity, positive definiteness and decrescency of $p(\cdot)$ on $R_{i} \times S(t)$, the definition of $\tau$ and compactness of $[\mathrm{t}, \tau]$ for any $t \in R_{0}$ imply

$$
\begin{gathered}
\left|\int_{\mathrm{t}}^{\tau} p\left[\sigma, \mathbf{x}\left(\sigma ; t_{0}, \mathbf{x}_{0}\right)\right] d \sigma\right|<\infty, \quad \forall\left(t, t_{0}, \mathbf{x}_{0}\right) \in R_{0} \times R_{i} \times N\left(t_{0}\right), \\
\left|\frac{\partial}{\partial t} \int_{t}^{\tau} \mathrm{p}\left[\sigma, \mathbf{x}\left(\sigma ; t_{0}, \mathbf{x}_{0}\right)\right] \mathrm{d} \sigma\right|=\left|\mathrm{p}\left[t, \mathbf{x}\left(t ; t_{0}, \mathbf{x}_{0}\right)\right]\right|<\infty, \quad \forall\left(t, t_{0}, \mathbf{x}_{0}\right) \in R_{0} \times R_{\imath} \times N\left(t_{0}\right),
\end{gathered}
$$

and

$$
\left\|\int_{\mathrm{t}}^{\tau} \operatorname{grad} p\left[\sigma, \mathbf{x}\left(\sigma ; t_{0}, \mathrm{x}_{0}\right)\right] d \sigma\right\|<\infty, \quad \forall\left(t, t_{0}, \mathrm{x}_{0}\right) \in R_{0} \times R_{i} \times N\left(t_{0}\right) .
$$

Now, (6.1)-(6.3), (6.6) and (6.7) yield

$$
\begin{gathered}
\left|v\left[t, \mathbf{x}\left(t ; t_{0}, \mathbf{x}_{0}\right)\right]\right|<\infty, \quad \forall\left(t, t_{0}, \mathbf{x}_{0}\right) \in R_{0} \times R_{i} \times N\left(t_{0}\right), \\
\left|\frac{\partial}{\partial t} v\left[t, \mathbf{x}\left(t ; t_{0}, \mathbf{x}_{0}\right)\right]\right|<\infty, \quad \forall\left(t, t_{0}, \mathbf{x}_{0}\right) \in R_{0} \times R_{i} \times N\left(t_{0}\right),
\end{gathered}
$$

and

$$
\left\|\operatorname{grad} v\left[t, \mathbf{x}\left(t ; t_{0}, \mathrm{x}_{0}\right)\right]\right\|<\infty, \quad \forall\left(t, t_{0}, \mathrm{x}_{0}\right) \in R_{0} \times R_{\imath} \times N\left(t_{0}\right)
$$


Let $t=t_{0}$ and $\mathrm{x}=\mathrm{x}_{0}$ be set in (6.8) Then,

$$
\begin{gathered}
|v(t, \mathbf{x})|<\infty, \quad \forall(t, \mathbf{x}) \in R_{\imath} \times N(t), \\
\left|\frac{\partial}{\partial \mathrm{t}} v(t, \mathbf{x})\right|<\infty, \quad \forall(t, \mathbf{x}) \in R_{\imath} \times N(t),
\end{gathered}
$$

and

$$
\|\operatorname{grad} v(t, \mathbf{x})\|<\infty, \quad \forall(t, \mathbf{x}) \in \mathrm{R}_{\mathbf{1}} \times \mathrm{N}(t)
$$

Differentiability of $p(\cdot)$ on $R_{\imath} \times S(t), S(t) \supseteq N(t),(66)$ and (6.9) prove

$$
v(t, \mathrm{x}) \in C^{(1)}\left[R_{\imath} \times N(t)\right]
$$

Invariance of both $D_{a}(t)$ and $N=D\left(R_{h}\right)$ on $R_{h}, D_{a}(t) \equiv D(t) \equiv N(t)$, continuity of $\mathbf{x}\left(t ; t_{0}, \mathrm{x}_{0}\right)$ in $\left(t ; t_{0}, \mathrm{x}_{0}\right) \in I_{0} \times R_{\imath} \times D\left(t_{0}\right)$, positive definiteness and decrescency of $p(\bullet)$ on $R_{\imath} \times N(t)$, $p(\bullet) \in L\left(R_{\imath}, S ; \mathrm{f}\right)$, (5.2), the definition of $\tau$ and compactness of $[t, \tau]$ guarantee existence of $\zeta_{2}(\bullet): R^{n} \mapsto R, \zeta_{1}(\mathbf{x}) \in C\left\{\cup\left[N(t): t \in R_{2}\right]\right\}$ and $\zeta_{2}(\mathbf{x}) \in C(N)$ such that

$$
\begin{gathered}
\zeta_{1}(0)=0, \quad i=1,2, \\
0<\zeta_{1}\left(\mathbf{x}_{0}\right) \leq \int_{t}^{\infty} \psi_{1}\left[\mathbf{x}\left(\sigma ; t_{0}, \mathbf{x}_{0}\right)\right] d \sigma, \quad \forall\left(\mathrm{t}, t_{0}, \mathbf{x}_{0} \neq 0\right) \in R_{0} \times R_{\imath} \times N\left(t_{0}\right), \\
\infty>\zeta_{2}\left(\mathbf{x}_{0}\right) \geq \int_{t}^{\tau} \psi_{2}\left[\mathbf{x}\left(\sigma ; t_{0}, \mathbf{x}_{0}\right)\right] d \sigma, \quad \forall\left(\mathrm{t}, t_{0}, \mathrm{x}_{0}\right) \in R_{0} \times R_{\imath} \times N,
\end{gathered}
$$

where $\psi_{\imath}(\cdot): R^{n} \mapsto R, i=1,2$, obey

$$
\begin{gathered}
\psi_{\imath}(\mathbf{x}) \in C\left\{\cup\left[N(t): t \in R_{1}\right]\right\} \quad \text { and } \quad \psi_{2}(\mathbf{x}) \in C(N), \\
\psi_{\imath}(0)=0, \quad i=1,2, \\
\psi_{1}(\mathbf{x})>0, \quad \forall(\mathbf{x} \neq 0) \in\left\{U\left[N(t): t \in R_{\imath}\right]\right\} \text { and } \psi_{2}(\mathbf{x})>0, \quad \forall(\mathbf{x} \neq 0) \in N, \\
\psi_{1}(\mathbf{x}) \leq p(t, \mathbf{x}), \quad \forall(t, \mathbf{x}) \in R_{\imath} \times N(t), \\
p(t, \mathbf{x}) \leq \psi_{2}(\mathbf{x}), \quad \forall(t, \mathbf{x}) \in R_{1} \times N .
\end{gathered}
$$

Such functions $\psi_{\mathrm{i}}(\bullet)$ exist due to decrescency and positive definiteness of $p(\bullet)$ on $R_{2} \times S(t)$ and $S(t) \supseteq N(t)$ Let $w_{\imath}(\cdot): R^{n} \mapsto R, w_{\imath}(\mathbf{x}) \in C\left(R^{n}\right), w_{\imath}(0)=0, i=1,2$, be such that

$$
\begin{gathered}
0<w_{1}(\mathbf{x}) \leq \zeta_{1}(\mathbf{x}), \quad \forall(\mathbf{x} \neq 0) \in \cup\left[N(t): t \in R_{\imath}\right], \\
w_{2}(\mathbf{x}) \geq \zeta_{2}(\mathbf{x})+w_{\mu}\left(\mathbf{x}_{\tau}\right), \quad \mathbf{x}_{\tau}=\mathbf{x}\left(\tau ; t_{0}, \mathbf{x}\right), \quad \forall\left(t_{0}, \mathbf{x}\right) \in R_{\imath} \times N,
\end{gathered}
$$

where $w_{\mu}(\cdot)$ is defined by (5.2) Now (5.2), (66) and (6.11)-(6 13) yield the following for $\left(t_{0}, \mathbf{x}_{0}\right)=(t, \mathbf{x})$ 


$$
\begin{gathered}
w_{1}(\mathbf{x}) \leq v(t, \mathbf{x}), \quad \forall(t, \mathbf{x}) \in R_{i} \times N(t), \\
v(t, \mathbf{x}) \leq w_{2}(\mathbf{x}), \quad \forall(t, \mathbf{x}) \in R_{i} \times N
\end{gathered}
$$

From $p(\bullet) \in L^{1}\left(R_{i}, S ; \mathrm{f}\right),(5.1 \mathrm{~b}),(6.10)$ and (6.14) it follows that a function $v(\bullet)$ defined by (5.1) is decrescent positive definite on $R_{i} \times N(t)$. Let it be assumed that there exist two such solutions $v_{1}\left(\bullet^{\circ}\right)$ and $v_{2}(\cdot)$ of $(6.1)$. Hence,

$v_{1}\left(t_{0}, \mathbf{x}_{0}\right)-v_{2}\left(t_{0}, \mathbf{x}_{0}\right)=\int_{t_{0}}^{\infty}\left\{p\left[\sigma, \mathbf{x}_{1}\left(\sigma ; t_{0}, \mathbf{x}_{0}\right)\right]-p\left[\sigma, \mathbf{x}_{2}\left(\sigma ; t_{0}, \mathbf{x}_{0}\right)\right]\right\} \mathrm{d} \sigma, \forall\left(t_{0}, \mathbf{x}_{0}\right) \in R_{2} \times N\left(t_{0}\right)$.

Uniqueness of the motions $\mathbf{x}\left(\bullet ; t_{0}, \mathrm{x}_{0}\right), \forall\left(t_{0}, \mathbf{x}\right) \in R_{1} \times S\left(t_{0}\right)$ (the Weak Smoothness Property), $S\left(t_{0}\right) \supseteq N\left(t_{0}\right)$ and uniqueness of $p(t, \mathbf{x})$ for every $(t, \mathbf{x}) \in R_{i} \times S(t)$ due to positive definiteness of $p(\bullet)$ on $S(t)$ imply

$$
\begin{aligned}
& \int_{t_{0}}^{\infty}\left\{p\left[\sigma, \mathbf{x}_{1}\left(\sigma ; t_{0}, \mathbf{x}_{0}\right)\right]-p\left[\sigma, \mathbf{x}_{2}\left(\sigma ; t_{0}, \mathbf{x}_{0}\right)\right]\right\} d \sigma \equiv \\
& \quad \int_{t_{0}}^{\infty}\left\{p\left[\sigma, \mathbf{x}\left(\sigma ; t_{0}, \mathrm{x}_{0}\right)\right]-p\left[\sigma, \mathbf{x}\left(\sigma ; t_{0}, \mathbf{x}_{0}\right)\right]\right\} \mathrm{d} \sigma=0, \forall\left(t_{0}, \mathbf{x}_{0}\right) \in R_{2} \times N\left(t_{0}\right) .
\end{aligned}
$$

This and (6.15) prove

$$
v_{1}\left(t_{0}, \mathrm{x}_{0}\right) \equiv v_{2}\left(t_{0}, \mathrm{x}_{0}\right)
$$

Hence, the function $v(\bullet)$ is the unique solution to (5). This completes the proof of necessity of the condition 4-a-1). Let $t_{0} \in R_{i}$ be arbitrary and $x_{k}, k=1,2, \ldots$, be a sequence converging to $u, x_{k} \mapsto u$ as $\mathrm{k} \mapsto \infty, \mathrm{x}_{\mathrm{k}} \in N\left(t_{0}\right)$ for all $\mathrm{k}=1,2, \ldots$, and $\mathrm{u} \in \partial N\left(t_{0}\right)$ in case $\partial N\left(t_{0}\right) \neq \phi$. Let $\rho \in R^{+}$be arbitrarily chosen so that $N(t) \supset \operatorname{ClP}_{\rho}(t)$ for all $t \in R_{i}$. Such $\rho$ exists because $p(\bullet)$ is positive definite and defines $\operatorname{ClP}_{\rho}(t)$, and because $\cap\left[N(t): t \in R_{i}\right]$ is a neighborhood of $\mathrm{x}=0$. Let $\tau_{k}$, $\tau_{k}=\tau\left(\mathbf{x}_{k}, \rho\right) \in R_{+}$, be the first instant satisfying (6.16),

$$
\mathbf{x}\left(t ; t_{0}, \mathbf{x}_{k}\right) \in \mathrm{ClP}_{\rho}(t), \quad \forall t \in\left[t_{0}+\tau_{k}, \infty[\right.
$$

Existence of such $\tau_{k}$ is ensured by $\mathrm{x}_{\mathrm{k}} \in N\left(t_{0}\right), N(t) \equiv D(t)$ and by the fact that $\cap\left[P_{\rho}(t): t \in R_{l}\right]$ is a neighborhood of $\mathrm{x}=0$ due to decrescency of $p(\bullet)$ on $R_{\imath} \times N(t)$ (Grujic et al. [12]). Continuity of the motions $\mathbf{x}\left(t ; t_{0}, \mathbf{x}\right)$ in $\left(t, t_{0}, \mathbf{x}\right) \in I_{0} \times R_{i} \times S\left(t_{0}\right)$ (the Weak Smoothness Property), $S\left(t_{0}\right) \supseteq D\left(t_{0}\right) \equiv N\left(t_{0}\right)$, positive invariance of $D(t)\left[(\mathrm{a})\right.$ of Lemma A.1] and $\mathrm{x}_{\mathbf{k}} \in N\left(t_{0}\right)$ imply

$$
\tau_{k} \mapsto \infty \text { as } \mathrm{k} \mapsto \infty
$$

Let $\mathrm{m} \in\{1,2, \ldots\}$ be such that $\mathrm{x}_{\mathrm{k}} \in\left[N\left(t_{0}\right)-\mathrm{ClP}_{\rho}\left(t_{0}\right)\right]$ for all $\mathrm{k}=\mathrm{m}, \mathrm{m}+1, \ldots$, which exists because $N\left(t_{0}\right)$ is open (Lemma 1), $N\left(t_{0}\right) \supset \mathrm{ClP}_{\rho}\left(t_{0}\right)$ and $\mathrm{x}_{\mathbf{k}} \mapsto \partial N\left(t_{0}\right)$ as $\mathrm{k} \mapsto \infty$. Let $\alpha$ be defined by

$$
\alpha=\min \left\{p(t, \mathbf{x}):(t, \mathbf{x}) \in R_{i} \times\left[S(t)-P_{\rho}(t)\right]\right\}
$$

Since $p(\bullet) \in L^{1}\left(R_{2}, S ; \mathrm{f}\right)$ then $\alpha \in R^{+}$. Hence, (6.16), (6.18) and the definitions of $\alpha$ and $\tau_{k}$ yield $v\left(t_{0}, \mathbf{x}_{\mathbf{k}}\right) \geq \alpha \tau_{k}, \forall t_{0} \in R_{h}$, which together with (6.17) proves necessity of the condition 4-a-ii). The fact: $N(t) \equiv D(t)$ implies the condition 4-a-iii) due to Definitions 1-3. The conditions under 4-b) follow from 4-a) due to (5.1), (5.3) and (5.5). This completes the proof of the necessity part. 
Sufficiency Let all the conditions of Theorem 1 hold and $t_{0} \in R_{1}$ be arbitrary Then $\mathrm{x}=0$ of the system (3.1) is uniformly asymptotically stable (Grujić et al [12], Hahn [15], Lakshmikantham and Leela [16], Miller and Michel [18], Zubov [20]). Hence, $\mathrm{x}=0$ has both $D\left(t_{0}\right)$ at $t_{0}$ and $D\left(R_{\imath}\right)$ (Definitions 13) so that $D_{a}\left(t_{0}\right)=D\left(t_{0}\right)$ and $D_{a}\left(R_{\imath}\right)=D\left(R_{\imath}\right)$ (Lemma A.2). Besides, $S\left(t_{0}\right) \supseteq N\left(t_{0}\right) \quad$ Since the function $\nu(\bullet)$ is solution to (5.1), [or, $\nu(\bullet)$ is solution to (5.3)], and it is positive definite and decrescent on $R_{\imath} \times N(t), p(\bullet) \in L^{1}\left(R_{1}, S ; \mathrm{f}\right)$, [or, $p(\bullet) \in E^{1}\left(R_{\imath}, S ; \mathrm{f}\right)$, and $p(\bullet)$ is decrescent positive definite on $R_{\imath} \times N(t)$, then $N\left(t_{0}\right) \equiv D\left(t_{0}\right)$ as shown in the proof of the necessity part. Hence, $D\left(R_{l}\right)=N$ (Definition 3), which completes the proof.

Conditions of Theorem 1 slightly change for the system (3.1) possessing the Weak Smoothness Property rather than the Strong Smoothness Property

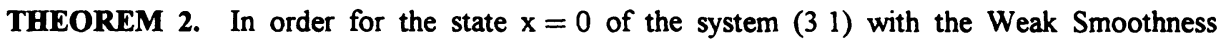
Property to have the domain $D\left(R_{l}\right)$ of uniform asymptotic stability on $R_{h}$, for a set $N\left(t_{0}\right)$, $N\left(t_{0}\right) \subseteq S\left(t_{0}\right)$ for all $t_{0} \in R_{\imath}$, to be the domain of its asymptotic stability at $t_{0} \in R_{h}: N\left(t_{0}\right) \equiv D\left(t_{0}\right)$, and for a set $N, N \subseteq S_{\imath}$, to be the domain of its uniform asymptotic stability on $R_{\imath}, N=D\left(R_{\imath}\right)$, it is both necessary and sufficient that

1) the set $N\left(t_{0}\right)$ is an open neighborhood of $\mathrm{x}=0$ for all $t_{0} \in R_{h}$,

2) the set $N$ is a connected neighborhood of $\mathrm{x}=0$,

3) $\mathbf{f}(t, \mathbf{x})=0$ for $(t, \mathrm{x}) \in R_{2} \mathrm{x} N(t)$ if and only if $\mathrm{x}=0$,

and

4) for any differentiable decrescent positive definite function $p(\cdot)$ on $R_{l} \times R^{n}$ obeying:

(a) $p(\bullet) \in L^{1}\left(R_{\imath}, R^{n} ; \mathrm{f}\right)$ the equations (5.1) have the unique solution function $v(\bullet)$ with the following properties

(i) $v(\bullet)$ is decrescent positive definite on $R_{\imath} \times N(t)$,

(ii) if the boundary $\partial N(t)$ of $N(t)$ is nonempty then $\mathrm{x} \mapsto \partial N(t), \mathrm{x} \in N(t)$, implies $v(t, \mathbf{x}) \mapsto \infty$ for every $t \in R_{\imath}$,

and

(iii) $N=\cap\left[N(t): t \in R_{1}\right]$,

or obeying

(b) $p(\bullet) \in E^{1}\left(R_{\imath}, R^{n} ; \mathrm{f}\right)$ the equations (5.3) have the unique solution function $v(\bullet)$ with the following properties.

(i) $v(\bullet)$ is decrescent positive definite on $R_{\imath} \times N(t)$,

(ii) if the boundary $\partial N(t)$ of $N(t)$ is nonempty then $\mathrm{x} \mapsto \partial N(t), \mathrm{x} \in N(t)$, implies $v(t, x) \mapsto 1$ for every $t \in R_{\imath}$,

and

(iii) $N=\cap\left[N(t): t \in R_{1}\right]$.

PROOF. Necessity. Let the system (3.1) possess the Strong Smoothness Property. Let $\mathrm{x}=0$ have the uniform asymptotic stability domain $D\left(R_{2}\right)$ on $R_{1}$ so that it has also the asymptotic stability domain $D\left(t_{0}\right)$ at every $t_{0} \in R_{2}$. Let $S\left(t_{0}\right) \supseteq D\left(t_{0}\right)$ and let $N\left(t_{0}\right)=D\left(t_{0}\right)$ for all $t_{0} \in R_{2}$ so that $D\left(R_{\imath}\right) \subseteq S$ and $N=D\left(R_{\imath}\right)$ Let a positive definite decrescent function $p(\cdot)$ on $R_{\imath} \times R^{n}$ be arbitrarily 
selected so that $p(\bullet) \in L^{1}\left(R_{\imath}, R^{n} ; \mathrm{f}\right)\left[p(\bullet) \in E^{1}\left(R_{i}, R^{n} ; \mathrm{f}\right)\right]$. From now on we should repeat the proof of necessity of the conditions of Theorem 1 in order to complete this proof.

Sufficiency. Let the system (3.1) possess the Weak Smoothness Property and let the conditions 1)4) hold. Hence, $x=0$ of the system is uniformly asymptotically stable (Grujić et al. [12], Hahn [15], Lakshmikantham and Leela [16], Miller and Michel [18], Zubov [20]) so that it has both the domain $D\left(R_{i}\right)$ of uniform asymptotic stability and the domain $D\left(t_{0}\right)$ of asymptotic stability at $t_{0} \in R_{4}$ (Definition 3). Let $\mathrm{x}_{0} \in\left[R^{n}-N\left(t_{0}\right)\right]$ and $t_{0}$ be arbitrary. Continuity of $\mathrm{x}\left(t ; t_{0}, \mathrm{x}_{0}\right)$ in $t \in R_{0}$ (the Weak Smoothness Property), positive definiteness of $p(\cdot)$ on $R_{i} \times R^{n}$ and the condition 4-a-ii), [4-b-ii)] imply $\mathbf{x}\left(t ; t_{0}, \mathrm{x}_{0}\right) \in\left[R^{n}-N(t)\right]$ for all $t \in I_{0}$. Therefore, $D\left(t_{0}\right) \subseteq C l N\left(t_{0}\right)$ and $D\left(R_{\imath}\right) \subseteq N$. Since $v(\bullet)$ is generated via (5.1) $[v(\bullet)$ is generated via (5.3)], then (as shown in the proof of the necessity part of Theorem 1) $v(t, \mathrm{x}) \mapsto \infty$ as $\mathrm{x} \mapsto \partial D(t), \mathrm{x} \in D(t)[v(t, \mathrm{x}) \mapsto 1$ as $\mathrm{x} \mapsto \partial D(t), \mathrm{x} \in D(t)]$, for every $t \in R_{i}$, which, together with the condition 4-a-1), [4-b-i)], proves $\partial D(t) \cap N(t)=\phi$ for every $t \in R_{i}$. This result, $D(t) \subseteq \mathrm{ClN}(t)$, and the fact that both $N(t)$ and $D(t)$ are open neighborhoods of $\mathrm{x}=0$ [condition 1 and Lemma A.1] imply $N(t)=D(t)$ and $N=D\left(R_{i}\right)$, which complete the proof.

Theorems 1 and 2 are based on the usage of $p(\bullet) \in L^{1}(\bullet),\left[\mathrm{p}(\bullet) \in \mathrm{E}^{1}(\cdot)\right]$. This means that $p(\bullet)$ should obey the condition 3) of Definition 4, [3) of Definition 5], which was used to generate $v(t, \mathrm{x}) \cdot \mapsto \infty$ as $\mathrm{x} \mapsto \partial N(t), \mathrm{x} \in N(t)[v(t, \mathrm{x}) \mapsto 1$ as $\mathrm{x} \mapsto \partial N(t), \mathrm{x} \in N(t)]$, for every $t \in R_{h}$, in order to determine exactly $D(t)$ and $D\left(R_{i}\right)$. If we are interested only in uniform asymptotic stability of $x=0$, then such a requirement need not be imposed on $p(\bullet)$ as explained in what follows:

THEOREM 3. In order for the state $x=0$ of the system (3.1) possessing the Weak Smoothness Property to be uniformly asymptotically stable on $R_{i}$ it is both necessary and sufficient that

1) $\mathrm{f}(t, 0)=0$ for all $t \in R_{i}$,

and

2) for any differentiable decrescent positive definite function $p(\cdot)$ on $R_{i}$ obeying the conditions 1) and 2) of Definition 4 the equations (5.1) have the unique solution function $v(\bullet)$ that is differentiable, decrescent and positive definite on $R_{i}$.

PROOF. Necessity. Let the system (3.1) possess the Weak Smoothness Property. Let $x=0$ be uniformly asymptotically stable on $R_{i}$ so that it has the domain $D\left(R_{i}\right)$ of uniform asymptotic stability (Definitions 1-3). Necessity of the condition 1) is proved in the same way as in the proof of Theorem 1. Since $D\left(R_{i}\right)$ and $S$ are neighborhoods of $\mathrm{x}=0$ then $D\left(R_{i}\right) \cap S \neq \phi$. Let $A$ be an open connected neighborhood of $\mathrm{x}=0$, which obeys $A \subseteq D\left(R_{i}\right) \cap S$, and let $p(\cdot)$ be arbitrary decrescent positive definite function on $R_{i} \times A$ obeying the conditions 1) and 2) of Definition 4. Hence, there exist positive definite functions $\psi_{i}(\bullet): R^{n} \mapsto R, i=1,2$, which satisfy (6.19),

$$
\psi_{1}(\mathbf{x}) \leq p(t, \mathbf{x}) \leq \psi_{2}(\mathbf{x}), \quad \forall(t, \mathbf{x}) \in R_{i} \times A
$$

From the conditions 1) and 2) of Definition 4 it results that there is a solution $v(\cdot)$ to (5.1), which is well defined and continuous on $\mathrm{ClB}_{\mu}$ and obeys (5.2). The set $L=A \cap B_{\mu}$ is also an open and connected neighborhood of $\mathrm{x}=0$ and $L \subseteq D\left(R_{i}\right)$. Let $\epsilon \in \mathrm{R}^{+}$be arbitrarily selected so that $B_{\epsilon} \subseteq L$. Hence, $B_{\epsilon} \subseteq D\left(R_{1}\right)$. Let $\rho \in \mathrm{R}^{+}$obeying $B_{\rho} \subseteq D_{s}(\epsilon)=\cap\left[D_{s}\left(t_{0}, \epsilon\right): t_{0} \in R_{l}\right]$ (Definitions 2 and 3 ), be arbitrarily selected. By following the proofs of $(6.9)$ and $(6.10)$ we prove that the function $v(\bullet)$ has the next property since $B_{\rho} \subseteq D_{s}(\epsilon) \subseteq B_{\epsilon} \subseteq L \subseteq A$,

$$
|v(t, \mathbf{x})|<\infty, \quad \forall(t, \mathbf{x}) \in R_{\imath} \times B_{\rho} .
$$


By following the proof of (6.14) we show that there are $w_{i}(\mathbf{x}) \in C\left(B_{\rho}\right), w_{i}(0)=0$ and $w_{i}(\mathbf{x})>0$, $\forall(\mathrm{x} \neq 0) \in B_{\rho}, i=1,2$, such that

$$
w_{i}(\mathbf{x}) \leq v(t, \mathbf{x}) \leq w_{2}(\mathbf{x}), \quad \forall(t, \mathbf{x}) \in R_{i} \times B_{\rho} .
$$

(6.20), (6.21), $w_{i}(\mathbf{x}) \in C\left(B_{\rho}\right)$, and $w_{i}(0)=0$ prove that the solution $v(\bullet)$ is decrescent positive definite on $R_{i} \times B_{\rho}$. Its uniqueness is proved in the same way as in the proof of the necessity part of Theorem 1. Hence, all the conditions are necessary for uniform asymptotic stability of $\mathrm{x}=0$ on $R_{h}$.

Sufficiency. Sufficiency of the conditions of Theorem 3 for uniform asymptotic stability of $x=0$ on $R_{i}$ of the system (3.1) is well known (Grujić et al. [12], Hahn [15], Lakshmikantham and Leela [16], Miller and Michel [18], Zubov [20]).

\section{EXAMPLES}

\section{Example 1.}

$$
\begin{aligned}
& \text { Let } \mathrm{x}=\left(x_{1} x_{2}\right)^{T} \in R^{2}, \mathrm{f}(\bullet)=\left[f_{1}(\bullet) f_{2}(\bullet)\right]^{T} \text { and } \\
& \qquad \begin{array}{l}
\frac{\mathrm{d} x_{1}}{\mathrm{~d} t}=\left[2\left(1+e^{-t}\right)\right]^{-1}\left\{e^{-t} x_{1}+\left[4-\left(1+e^{-t}\right) x_{1}^{2}\right]^{2}\left[-\left(5+e^{-t} \sin t\right) x_{1}+x_{2}\right]\right\} \\
\frac{\mathrm{d} x_{2}}{\mathrm{~d} t}=\left[2\left(1+e^{-t}\right)\right]^{-1}\left\{e^{-t} x_{2}-\left[10-\left(1+e^{t}\right) x_{2}^{2}\right]\left[4 x_{1}+\left(1+t^{2}\right)\left(1+2 t^{2}\right)^{-1} x_{2}\right]\right\} .
\end{array}
\end{aligned}
$$

The system possesses the Weak Smoothness Property on $R_{i} \times R^{2}$, where $\left.R_{i}=\right]-1, \infty[$ and $S=R^{2}$. The set $S_{e}$ of the equilibrium states is singleton, $S_{e}=\{0\}$. The function $v(\bullet)(7.2)$,

$$
v(t, \mathbf{x})=\frac{\left(1+e^{-t}\right) x_{1}^{2}}{4-\left(1+e^{-t}\right) x_{1}^{2}}-\ln \left[\frac{10-\left(1+e^{-t}\right) x_{2}^{2}}{10}\right],
$$

and the function $p(\bullet)(7.3)$,

$$
p(t, \mathrm{x})=4\left(5+e^{-t} \sin t\right) x_{1}^{2}+\left(1+t^{2}\right)\left(1+2 t^{2}\right)^{-1} x_{2}^{2},
$$

satisfy the equations (5.1). For the function $p(\bullet)(7.3)$ we find that $\psi_{1}\left(\bullet^{\circ}\right.$ and $\psi_{2}\left({ }^{\circ}\right)$, $\psi_{1}(\mathbf{x})=\left(x_{1}^{2}+2^{-1} x_{2}^{2}\right)$ and $\psi_{2}(\mathbf{x})=\left(32 x_{1}^{2}+x_{2}^{2}\right)$, satisfy

$$
\begin{aligned}
& \psi_{1}(\mathbf{x}) \leq p(t, \mathbf{x}), \quad \forall(t, \mathbf{x}) \in R \times R^{2}, \\
& p(t, \mathbf{x}) \geq \psi_{2}(\mathbf{x}), \quad \forall(t, \mathbf{x}) \in R \times R^{2} .
\end{aligned}
$$

Similarly, for the function $v(\bullet) \quad(7.2)$ we find that $w_{1}(\bullet)$ and $w_{2}(\bullet)$, where $w_{1}(\mathbf{x})=\left\{\left[x_{1}^{2}\left(4-x_{1}^{2}\right)^{-1}\right]-\ln \left[\left(10-x_{2}^{2}\right) 10^{-1}\right]\right\} \quad$ and $\quad w_{2}(x)=\left\{4 x_{1}^{2}\left[4-(1+e) x_{1}^{2}\right]^{-1}-\ln [10-\right.$ $\left.\left.(1+e) x_{2}^{2}\right] 10^{-1}\right\}$, obey

where

$$
\begin{gathered}
w_{1}(\mathbf{x}) \leq v(t, \mathbf{x}), \quad \forall(t, \mathbf{x}) \in R_{i} \times N(t), \\
\nu(t, \mathbf{x}) \geq w_{2}(\mathbf{x}), \quad \forall(t, \mathbf{x}) \in R_{i} \times N,
\end{gathered}
$$

and

$$
N(t)=\left\{\mathrm{x}: \mathrm{x} \in R^{2}, x_{1}^{2}<4\left(1+e^{-t}\right)^{-1}, x_{2}^{2}<10\left(1+e^{-t}\right)^{-1}\right\}
$$

$$
N=\left\{\mathrm{x}: \mathrm{x} \in R^{2}, x_{1}^{2}<4(1+e)^{-1}, x_{2}^{2}<10(1+e)^{-1}\right\}
$$


The functions $v(\cdot)(7.2)$ and $p(\bullet)(7.3)$ are differentiable. We may now conclude that $p(\bullet) \in L^{1}\left(R_{i}, S, f\right)$. For such $p(\bullet)$ the function $v(\bullet)(7.3)$ and the set $N(t)(7.7)$, as well as the system (7.1) itself, fulfill all the conditions of Theorem 2. Hence, $x=0$ of the system (7.1) is uniformly asymptotically stable with the domain $D(t)$ of asymptotic stability: $D(t)=N(t)(7.6)$ on $R_{i}$ and with the domain $D$ of uniform asymptotic stability on $R_{i}: D=N(7.7)$. Simulation results are shown in Fig. 1a,b. They illustrate an influence of the initial time $t_{0}$ on $D\left(t_{0}\right)$ and on system solutions with the same initial state at different initial instants. They illustrate also an influence of the initial state $x_{0}$ on system solutions at every initial time $t_{0} \in\{0 s, 0.3 s\}$. The initial states $x_{0}=\left(\begin{array}{ll}1.4 & 2.236\end{array}\right)^{T} \in D\left(t_{0}\right)$ and $\mathrm{x}_{0}=\left(\begin{array}{ll}-1.42 .236\end{array}\right)^{T} \in D\left(t_{0}\right)$ for every $t_{0} \in\{0 s, 0.3 s\}$. The initial state $\mathrm{x}_{0}=\left(\begin{array}{ll}1.42 & 2.236\end{array}\right)^{T}$ is in $D\left(t_{0}\right)$ for $t_{0}=0.3 s$, but not for $t_{0}=0 s$.

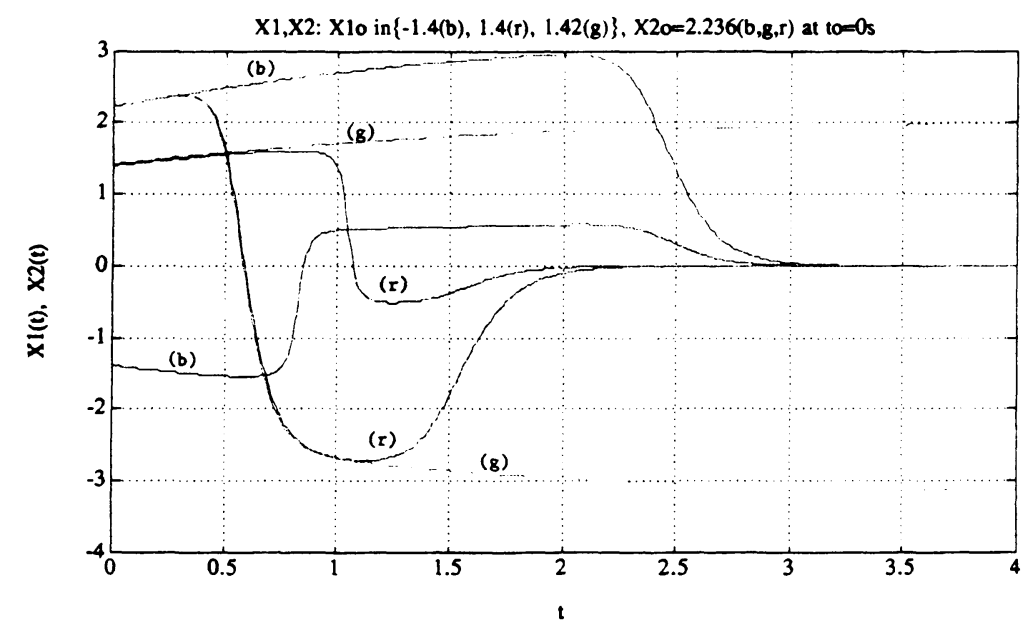

Fig. 1: a) $x_{01}=\left(\begin{array}{ll}-1.42 .236\end{array}\right)^{T} \in D(0)$ and $x_{02}=\left(\begin{array}{ll}1.4 & 2.236\end{array}\right)^{T} \in D(0)$ but $\mathrm{x}_{03}=\left(\begin{array}{ll}1.42 & 2.236\end{array}\right)^{T} \notin D(0)$.

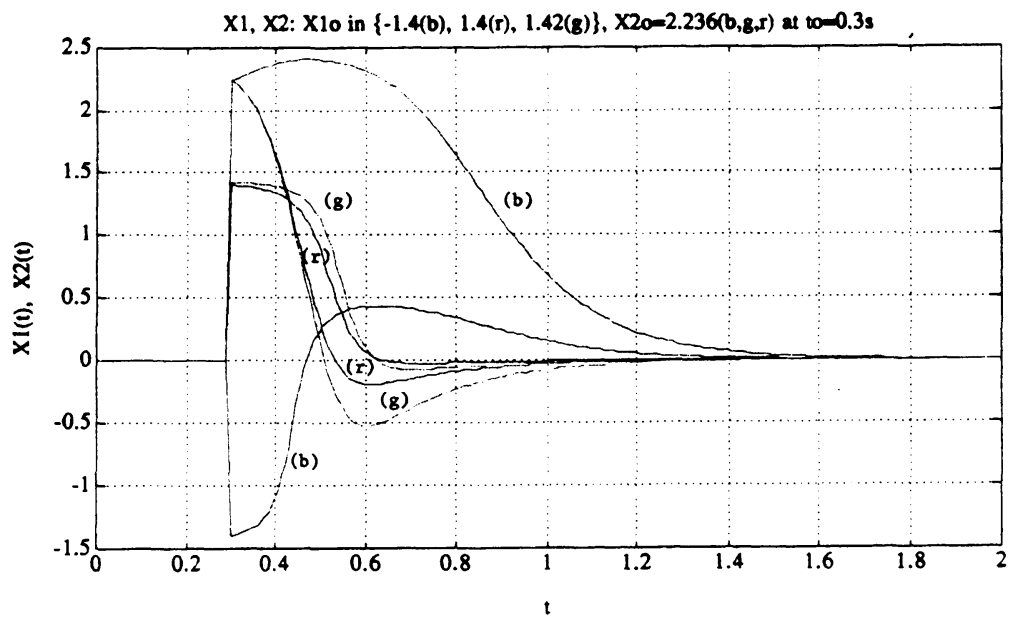

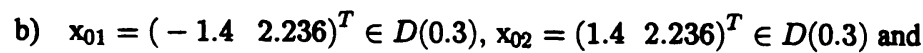
$\mathrm{x}_{03}=\left(\begin{array}{ll}1.42 & 2.236\end{array}\right)^{T} \in D(0.3)$. 


\section{Example 2.}

$$
\begin{aligned}
& \text { Let } \mathbf{x}=\left(\begin{array}{lll}
x_{1} & x_{2} & x_{3}
\end{array}\right)^{T} \in R^{3}, \mathbf{f}(\bullet)=\left[f_{1}(\bullet) f_{2}(\bullet) f_{3}(\bullet)\right]^{T} \text { and } \\
& \qquad \frac{\mathbf{d x}}{\mathrm{d} t}=-\frac{\cos \mathrm{t}+(2+\cos \mathrm{t})\left[99-(2+\sin t) \mathbf{x}^{T} \mathbf{H x}\right]^{2}}{2(2+\sin \mathrm{t})} \mathbf{x}=\mathbf{f}(t, \mathbf{x}), \\
& H=\left[\begin{array}{rrr}
2 & -1 & 2 \\
-1 & 4 & 0 \\
2 & 0 & 6
\end{array}\right] .
\end{aligned}
$$

The matrix $\mathrm{H}$ is symmetric and positive definite. The system (7.8) has the Weak Smoothness Property with $S=R^{3}$ and has the single equilibrium state at $\mathrm{x}=0$ so that $\mathrm{f}(t, \mathrm{x})=0$ for all $t \in R$ iff $\mathrm{x}=0$. For the function $p(\cdot)$,

$$
p(t, \mathbf{x})=99(2+\operatorname{cost}) \mathbf{x}^{T} \mathrm{Hx}
$$

the function $v(\cdot)$,

$$
v(t, \mathrm{x})=\frac{2(2+\sin t) \mathrm{x}^{T} \mathrm{Hx}}{99-(2+\sin t) \mathrm{x}^{T} \mathrm{Hx}}
$$

satisfies (5.1). The following comparison functions are found for them,

$$
\begin{aligned}
& \psi_{1}(\mathbf{x})=99 \mathrm{x}^{T} \mathrm{Hx} \\
& \psi_{2}(\mathbf{x})=297 \mathbf{x}^{T} \mathrm{Hx} \\
& w_{1}(\mathbf{x})=\mathrm{x}^{T} \mathrm{Hx}\left(99-\mathrm{x}^{T} \mathrm{Hx}\right)^{-1} \\
& w_{2}(\mathbf{x})=3 \mathbf{x}^{T} \mathrm{Hx}\left(99-3 \mathbf{x}^{T} \mathrm{Hx}\right)^{-1}
\end{aligned}
$$

so that

$$
\begin{gathered}
\psi_{1}(\mathbf{x}) \leq p(t, \mathbf{x}) \leq \psi_{2}(\mathbf{x}), \quad \forall(t, \mathbf{x}) \in R \times R^{3}, \\
w_{1}(\mathbf{x}) \leq v(t, \mathbf{x}), \quad \forall(t, \mathbf{x}) \in R \times N(t),
\end{gathered}
$$

and

$$
w_{2}(\mathbf{x}) \geq v(t, \mathbf{x}), \quad \forall(t, \mathbf{x}) \in R \times N
$$

where

$$
N(t)=\left\{\mathrm{x}: \mathrm{x} \in R^{3}, \mathrm{x}^{T} \mathrm{Hx}<\frac{99}{2+\sin t}\right\} \equiv \operatorname{In} N(t) ; \quad 0 \in N(t), \quad \forall t \in R,
$$

and

$$
N=\left\{\mathrm{x}: \mathrm{x} \in R^{3}, \mathrm{x}^{T} \mathrm{Hx}<33\right\}
$$

The system (7.8), the functions $p(\cdot)(7.9)$ and $v(\cdot)(7.10)$, and the set $N(t)$ satisfy all the conditions of Theorem 2 for $R_{i}=R$. The equilibrium state $\mathrm{x}=0$ of the system is uniformly asymptotically stable with 


$$
D(t)=\left\{\mathrm{x}: \mathrm{x} \in R^{3}, \mathrm{x}^{T} \mathrm{Hx}<\frac{99}{2+\sin t}\right\}, \forall t \in R
$$

and

$$
D=\left\{\mathrm{x}: \mathrm{x} \in R^{3}, \mathrm{x}^{T} \mathrm{Hx}<33\right\}
$$

The simulations completely verified the above results. They are shown for two initial states, $\mathrm{x}_{01}=(6.6$ $1-2)^{T}$, Fig. 2, and $x_{02}=\left(\begin{array}{lll}7.96 & 1 & -2\end{array}\right)^{T}$ that was preserved unchanged at three different initial instants $t_{0} \in\{0 s, 3.893 \mathrm{~s}, 3.895 \mathrm{~s}\}$, Fig. 3a,b,c. In this example, $x_{01}=\left(\begin{array}{lll}6.6 & 1-2\end{array}\right)^{T} \in D(0)$, Fig. 2. However, $\mathrm{x}_{02} \notin D(0)$, but $\mathrm{x}_{02} \in D(3.893) \cap D(3.895)$, Fig. 3a,b,c.

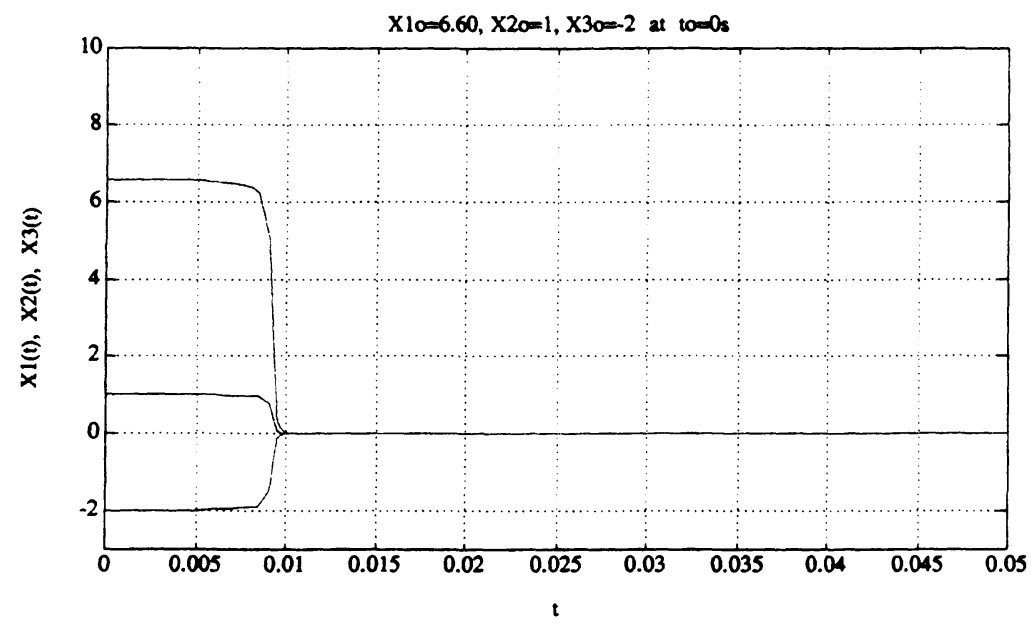

Fig. 2: $x_{01}=\left(\begin{array}{lll}6.6 & 1 & -2\end{array}\right)^{T} \in D(0)$.

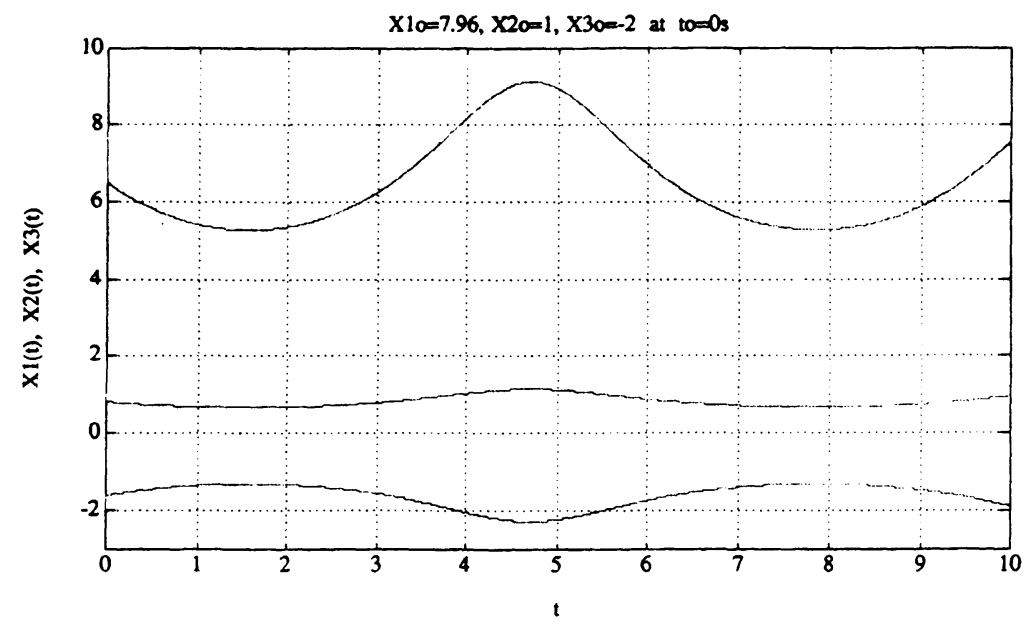

Fig. 3: a) $\quad x_{02}=\left(\begin{array}{lll}7.96 & 1 & -2\end{array}\right)^{T} \notin D(0)$. 


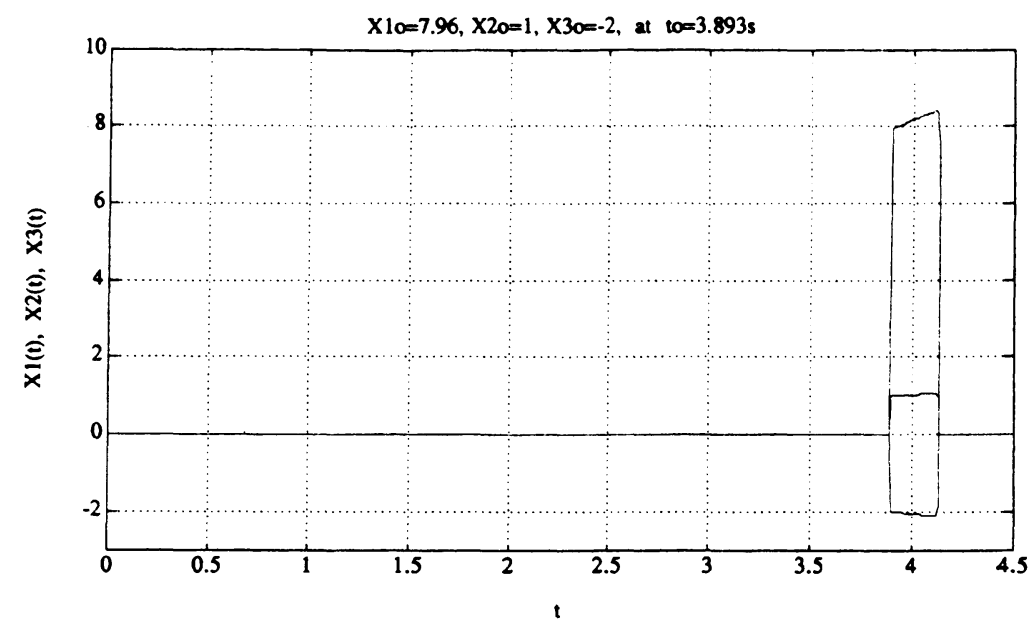

Fig. 3: b) $\mathrm{x}_{02}=\left(\begin{array}{lll}7.96 & 1 & -2\end{array}\right)^{T} \in D(3.893)$.

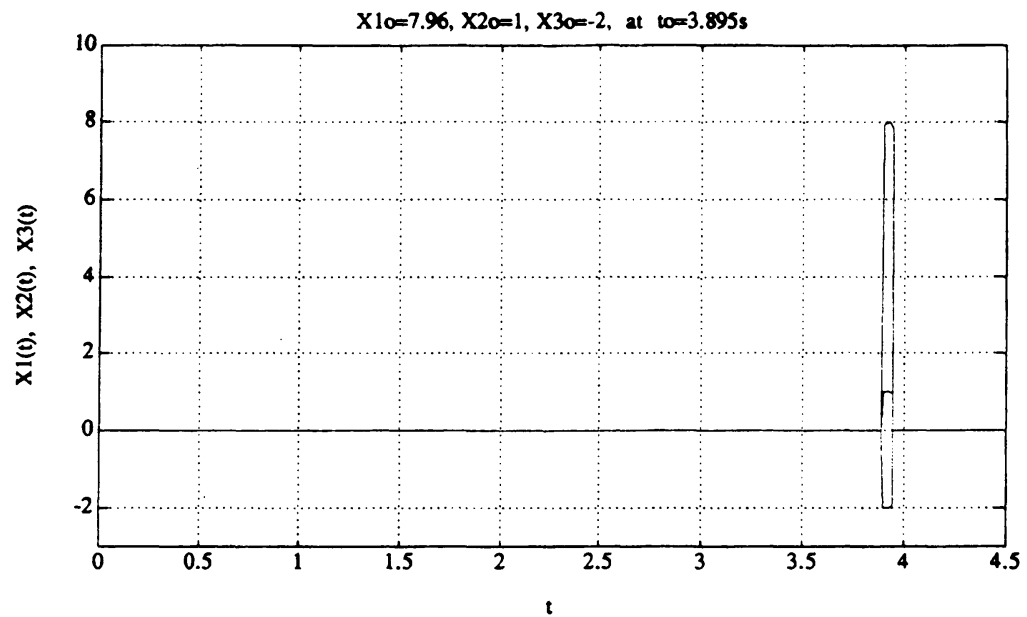

Fig. 3: c) $\quad x_{02}=\left(\begin{array}{lll}7.96 & 1 & -2\end{array}\right)^{T} \in D(3.895)$. The rate of convergence is much higher than when $t_{0}=3.893 \mathrm{~s}$.

\section{Example 3.}

$$
\begin{aligned}
& \text { Let } \mathrm{x}=\left(x_{1} x_{2}\right)^{T} \in R^{2}, \mathrm{f}(\bullet)=\left[f_{1}(\bullet) f_{2}(\cdot)\right]^{T} \text { and } \\
& \qquad \frac{\mathrm{d} x_{1}}{\mathrm{~d} t}=\frac{1}{\left(1+2 t^{2}\right)\left[1+t^{2}-4\left(1+2 t^{2}\right) x_{2}^{2}\right]}\left\{-t-\left[1+t^{2}-\left(1+2 t^{2}\right)\left(x_{1}^{2}+2 x_{2}^{2}\right)\right]^{2}\right\} x_{1}, \quad \text { (7.11a) } \\
& \qquad \frac{\mathrm{d} x_{2}}{\mathrm{~d} t}=\frac{1}{\left(1+2 t^{2}\right)\left[1+t^{2}-2\left(1+2 t^{2}\right) x_{1}^{2}\right]}\left\{-t+\left[1+t^{2}-\left(1+2 t^{2}\right)\left(x_{1}^{2}+2 x_{2}^{2}\right)\right]^{2}\right\} x_{2}, \quad \text { (7.11b) }
\end{aligned}
$$

The system (7.11) has the Weak Smoothness Property with

$$
S(t)=\left\{\mathrm{x}: \mathrm{x} \in R^{2}, x_{1}^{2}<\left(1+t^{2}\right)\left[2\left(1+2 t^{2}\right)\right]^{-1}, x_{2}^{2}<\left(1+t^{2}\right)\left[4\left(1+2 t^{2}\right)\right]^{-1}\right\} .
$$

The system has the unique equilibrium state $\mathrm{x}=0$. The function $p(\cdot)$, 


$$
\begin{aligned}
& p(t, \mathbf{x}) \equiv p(\mathbf{x})=2\left(x_{1}^{2}+2 x_{2}^{2}\right), \text { and the function } v(\bullet) \\
& \nu(t, \mathbf{x})=\frac{\left(1+2 t^{2}\right)\left(x_{1}^{2}-2 x_{2}^{2}\right)}{1+t^{2}-\left(1+2 t^{2}\right)\left(x_{1}^{2}+2 x_{2}^{2}\right)}
\end{aligned}
$$

show that $p(\bullet) \in L^{1}(R, S, \mathrm{f})$ so that we may apply Theorem 3 . Since the solution function $v(\bullet)$ to $(5.1)$ is not positive definite then Theorem 3 is not satisfied. Hence, $x=0$ is not uniformly asymptotically stable. Simulation results shown in Fig. $4 a, b$ will illustrate that $x=0$ is not uniformly asymptotically stable. This example illustrates importance and usefulness of the necessity of the conditions of Theorem 3 , as well as of Theorems 1 and 2 . Since it is not satisfied there is not any sense to try constructing a more suitable function $v(\cdot)$ in order to prove uniform asymptotic stability of $\mathrm{x}=0$ because it is impossible. This example illustrates the usefulness of the single step construction of the function $v(\bullet)$ by using any of the above theorems.
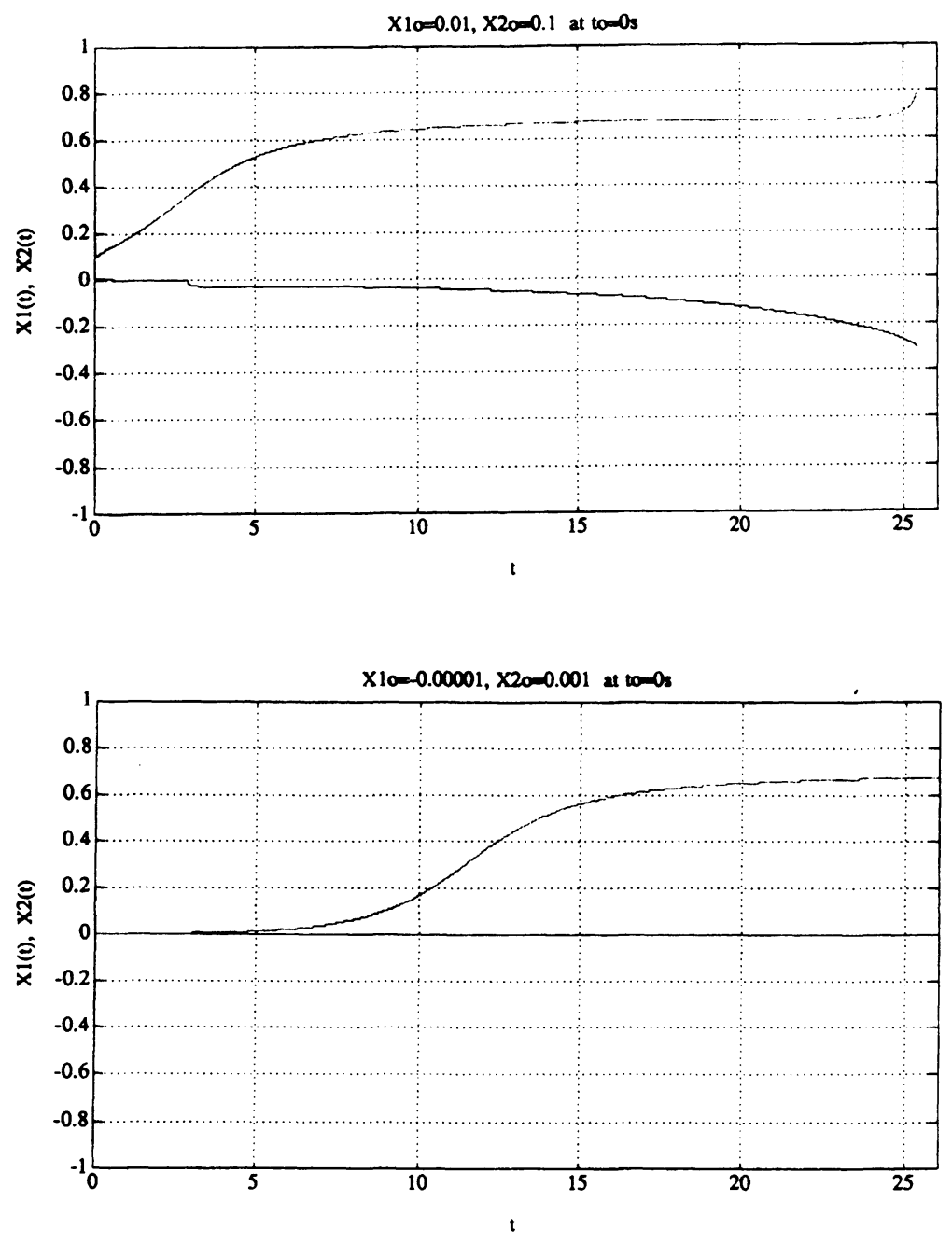

Fig. 4: Motions for two different small initial conditions under a) and b) express instability of $\mathrm{x}=0$ at $t_{0}=0 \mathrm{~s}$. 


\section{CONCLUSION}

The new methodology for a single simultaneous exact construction of a system Lyapunov function and accurate determination of the domain of asymptotic stability of $x=0$ established in [4]-[11] for time invariant systems is essentially broadened to time-varying systems The conditions are presented as both necessary and sufficient, and in terms of arbitrary choice of a differentiable decrescent positive definite function $p(\bullet) \in L^{1}(\bullet),\left\{\right.$ or, $\left.p(\bullet) \in E^{1}(\cdot)\right\}$, and in terms of properties of a solution function $v(\bullet)$ to $v^{\prime}(\bullet)=-p(\bullet)(5.1),\left\{\right.$ or in terms of properties of a solution function $v(\bullet)$ to $v^{\prime}(\bullet)=-[1-v(\bullet)] p(\bullet)$ (5.3)\}, respectively, obtained for arbitrarily selected function $p(\bullet)$. The families $L^{1}(\bullet)$ and $E^{1}(\bullet)$ are determined by Definitions 4 and 5 If so obtained $v(\bullet),\{v(\bullet)\}$, is also differentiable decrescent positive definite then (Theorem 3) $\mathrm{x}=0$ IS uniformly asymptotically stable If $v(\bullet),\{v(\bullet)\}$, does not have any of these properties then $\mathrm{x}=0$ IS NOT uniformly asymptotically stable The solution to the problem of uniform asymptotic stability is obtained under a single application of Theorem 3 The same conclusion is valid for the determination of the domain of (uniform) asymptotic stability of $x=0$ via Theorems 1 and 2 Numerous simulations were carried out They completely verified the theoretical results. Few of them are presented in the paper

\section{APPENDIX}

LEMMA A.1. Let the system (3.1) possess the Weak Smoothness Property and let $\mathrm{x}=0$ be uniformly attractive on $R_{l}$ with the instantaneous domain $D_{a}(t)$ of attraction obeying $D_{a}(t) \subseteq S(t)$ for all $t \in R_{1}$ and with the domain $D_{a}\left(R_{1}\right)$ of uniform attraction on $R_{2}$.

a) If $R_{\imath} \subset R$ then

1) $\left(t_{0}, \mathbf{x}_{0}\right) \in R_{\imath} \times D_{a}\left(t_{0}\right)$ implies $\mathbf{x}\left(t ; t_{0}, \mathbf{x}_{0}\right) \in D_{a}(t)$ for all $t \in R_{\imath}$, that is that $D_{a}(t)$ is invariant on $R_{h}$,

2) $D_{a}(t)$ is an open neighborhood of $\mathrm{x}=0$ at any $t \in R_{\imath}: D_{a}(t) \equiv \operatorname{In} D_{a}(t)$, and

3) $D_{a}\left(R_{1}\right)$ is a connected neighborhood of $\mathrm{x}=0$ such that $\left(t_{0}, \mathrm{x}_{0}\right) \in R_{1} \times D_{a}\left(R_{1}\right)$ implies $\mathbf{x}\left(t ; t_{0}, \mathrm{x}_{0}\right) \in D_{a}\left(R_{l}\right)$ for every $t \in R_{i}$, that is that $D_{a}\left(R_{l}\right)$ is invariant on $R_{l}$ in case $D_{a}(t)=D_{a}\left(R_{l}\right)$ for all $t \in R_{\imath}$ Otherwise, $D_{a}\left(R_{l}\right)$ is connected neighborhood of $x=0$

b) If $R_{1}=R$ then

1) $D_{a}(t)$ is invariant, that is that $\left(t_{0}, \mathrm{x}_{0}\right) \in R \times D_{a}\left(t_{0}\right)$ implies $\mathbf{x}\left(t ; t_{0}, \mathrm{x}_{0}\right) \in D_{a}(t)$ for all $t \in R$,

2) $D_{a}(t)$ is an open neighborhood of $\mathrm{x}=0$ at any $t \in R: D_{a}(t) \equiv \operatorname{In} D_{a}(t)$, and

3) $D_{a}$ is an invariant connected neighborhood of $\mathrm{x}=0:\left(t_{0}, \mathrm{x}_{0}\right) \in R \times D_{a}(R)$ implies $\mathbf{x}\left(t ; t_{0}, \mathbf{x}_{0}\right) \in D_{a}(R)$ for all $t \in R$ in case $D_{a}(t)=D_{a}(R)$ for all $t \in R$ Otherwise, $D_{a}=D_{a}(R)$ is connected neighborhood of $x=0$.

PROOF. Let the system (3.1) possess the Weak Smoothness Property and let $\mathrm{x}=0$ be uniformly attractive on $R_{l}$ with the instantaneous domain $D_{a}(t)$ of attraction obeying $D_{a}(t) \subseteq S(t)$ for all $t \in R_{\imath}$ and with the domain $D_{a}\left(R_{\imath}\right)$ of uniform attraction on $R_{\imath}$. Hence, $D_{a}\left(R_{\imath}\right)=\cap\left[D_{a}\left(t_{0}\right): t_{0} \in R_{l}\right]$ (Definition 1).

a) Let $t_{0}$ and $\tau_{0} \in R_{2}, t_{0} \neq \tau_{0}$. Let $\mathbf{x}^{*}=\mathbf{x}\left(\tau_{0} ; t_{0}, \mathrm{x}_{0}\right)$ for any $\mathbf{x}_{0} \in D_{a}\left(t_{0}\right)$ Then, $\mathbf{x}\left(t ; t_{0}, \mathbf{x}_{0}\right) \rightarrow 0$ as $t \rightarrow \infty$ Since $\mathbf{x}\left(t ; \tau_{0}, \mathbf{x}^{*}\right) \equiv \mathbf{x}\left[t ; \tau_{0}, \mathbf{x}\left(\tau_{0} ; t_{0}, \mathbf{x}_{0}\right)\right]=\mathbf{x}\left(t ; t_{0}, \mathbf{x}_{0}\right)$ due to (i) of the Weak Smoothness Property and $D_{a}\left(t_{0}\right) \subseteq S\left(t_{0}\right)$ then $\mathbf{x}\left(t ; \tau_{0}, \mathbf{x}^{*}\right) \rightarrow 0$ as $t \rightarrow \infty$. Hence, $\mathbf{x}^{*}=\mathbf{x}\left(\tau_{0} ; t_{0}, \mathbf{x}_{0}\right) \in D_{a}\left(\tau_{0}\right)$ that proves the statement under a-1). Let $\zeta \in R^{+}$be such that $B_{2 \zeta} \subset D_{a}\left(R_{h}\right)$. Let there exist $t_{0}^{\prime} \in R_{\imath}$ and $x_{0}^{\prime} \in \partial D_{a}\left(t_{0}^{\prime}\right) \cap D_{a}\left(t_{0}^{\prime}\right)$. Let $\left.\epsilon \in\right] 0, \zeta / 2[$. Then, (i) of the Weak Smoothness Property, $D_{a}\left(t_{0}^{\prime}\right) \subseteq S\left(t_{0}^{\prime}\right)$ and (a) of Definition 1 imply existence of $\rho \in R^{+}, \rho=\rho\left(t_{0}^{\prime}, \mathrm{x}_{0}, \epsilon\right)$, 
such that $\left\|\mathrm{x}_{0}-\mathrm{x}_{0}^{\prime}\right\|<\rho$ ensures $\left\|\mathbf{x}\left(t_{0}^{\prime}+2 \sigma^{\prime} ; t_{0}^{\prime}, \mathrm{x}_{0}\right)-\mathbf{x}\left(t_{0}^{\prime}+2 \sigma^{\prime} ; t_{0}^{\prime}, \mathrm{x}_{0}^{\prime}\right)\right\|<\epsilon$, where $\sigma^{\prime}=\tau\left(t_{0}^{\prime}, \mathrm{x}_{0}^{\prime}, \zeta\right)$ (Definition 1). Since $\epsilon<\zeta / 2$ and $\left\|\mathbf{x}\left(t_{0}^{\prime}+2 \sigma^{\prime} ; t_{0}^{\prime}, \mathrm{x}_{0}^{\prime}\right)\right\|<\zeta$ then $\mathbf{x}\left(t_{0}^{\prime}+2 \sigma^{\prime} ; t_{0}^{\prime}, \mathrm{x}_{0}\right) \in B_{2 \zeta} \subset D_{a}\left(R_{i}\right)$. Hence, $\mathrm{x}_{0} \in D_{a}\left(t_{0}^{\prime}\right)$. However, $\mathrm{x}_{0}$ may be chosen in a $\rho-$ neighborhood of $x_{0}^{\prime}$ out of $D_{a}\left(t_{0}^{\prime}\right)$ that is contradicted by the obtained $x_{0} \in D_{a}\left(t_{0}^{\prime}\right)$. Since the former is true then the latter is wrong so that there are not $t_{0}^{\prime} \in R_{i}$ and $x_{0}^{\prime} \in \partial D_{a}\left(t_{0}^{\prime}\right) \cap D_{a}\left(t_{0}^{\prime}\right)$. Hence, if $x_{0}^{\prime} \in \partial D_{a}\left(t_{0}^{\prime}\right)$ then $x_{0}^{\prime} \notin D_{a}\left(t_{0}^{\prime}\right)$. The set $D_{a}\left(t_{0}\right)$ is open for all $t_{0} \in R_{\imath}$ and it is neighborhood of $\mathrm{x}=0$ due to Definition 1 . Hence, the statement under a-2) is correct. Furthermore, $D_{a}\left(R_{i}\right)$ is a neighborhood of $\mathrm{x}=0$ by definition (Definition 1). Its connectedness is proved as follows. Let us assume that it is not connected. Then, there are disjoint sets $D_{a i}$, $i=1,2, \ldots, N$, such that $D_{a}\left(R_{i}\right)=\cup\left[D_{a i}: i=1,2, \ldots, N\right]$. One of $D_{a i}$ is not a neighborhood of $\mathbf{x}=0$. Let it be $D_{a 1}$. Then $\mathrm{x}_{0} \in D_{a 1}$ implies $\mathbf{x}\left(t ; t_{0}, \mathrm{x}_{0}\right) \rightarrow 0$ as $t \rightarrow \infty, \forall t_{0} \in R_{i}$. Hence, there is $t_{1} \in R_{0}$ such that $\mathbf{x}\left(t_{1} ; t_{0}, \mathbf{x}_{0}\right) \notin D_{a}\left(R_{i}\right)$ because of continuity of $\mathbf{x}\left(t ; t_{0}, \mathbf{x}_{0}\right)$ in $t \in R_{0}$, $\forall t_{0} \in R_{i}$, and because $D_{a 1}$ is disjoint subset of $D_{a}\left(R_{i}\right)$ that is not a neighborhood of $\mathrm{x}=0$, which is impossible due to $\mathbf{x}\left[t ; t_{1}, \mathbf{x}\left(t_{1} ; t_{0}, \mathbf{x}_{0}\right)\right]=\mathbf{x}\left(t ; t_{0}, \mathbf{x}_{0}\right) \rightarrow 0$ as $t \rightarrow \infty$. Hence, the assumption on disconnectedness of $D_{a}\left(R_{i}\right)$ is incorrect. In order to prove that $\left(t_{o}, \mathrm{x}_{0}\right) \in R_{i} \times D_{a}\left(R_{i}\right)$ implies $\mathbf{x}\left(t ; t_{0}, \mathrm{x}_{0}\right) \in D_{a}\left(R_{i}\right)$ for all $t \in R_{i}$ let $\left(t_{o}, \mathrm{x}_{0}\right) \in R_{i} \times D_{a}\left(R_{i}\right)$ be arbitrarily selected. The condition b-4) of Definition 1 guarantees $\sup \left[\tau_{m}\left(t_{o}, x_{0}, \zeta\right): t_{0} \in R_{i}\right]=\alpha<\infty, \forall \zeta \in R^{+}$. Let $t_{2} \in R_{i}$ be arbitrary. Evidently, $\beta=t_{o}-t_{2}$ obeys $|\beta|=\left|t_{o}-t_{2}\right|<\infty$. Let $\mathbf{x}_{2}=\mathbf{x}\left(t_{2} ; t_{0}, \mathrm{x}_{0}\right)$ so that $\mathbf{x}_{2} \in D_{a}\left(t_{2}\right)$ and $\sup \left[\tau_{m}\left(t_{2}, \mathrm{x}_{2}, \zeta\right): t_{0} \in R_{i}\right]=\sup \left[\tau_{m}\left(t_{0}, \mathrm{x}_{0}, \zeta\right)+\beta: t_{0} \in R_{l}\right]=\alpha+\beta<\infty$ that proves $x_{2}=x\left(t_{2} ; t_{0}, x_{0}\right) \in D_{a}\left(R_{i}\right)$ in view of the condition b) of Definition 1 and $\mathbf{x}\left(t ; t_{2}, x_{2}\right)=\mathbf{x}\left(t ; t_{0}, x_{0}\right)$. This completes the proof of all the statements under $\left.a\right)$.

b) The assertions under b) directly follow from those under a) in case $R_{i}=R$.

\section{LEMMA A.2.}

(a) If the state $x=0$ of the system (3.1) possessing the Weak Smoothness Property is asymptotically stable and its domain $D_{a}\left(t_{0}\right)$ of attraction at $t_{0} \in R_{i}$ obeys $D_{a}\left(t_{0}\right) \subseteq S\left(t_{0}\right)$ for all $t_{0} \in R_{i}$ then its domains $D_{a}\left(t_{0}\right), \quad D_{s}\left(t_{0}\right)$ and $D\left(t_{0}\right)$ are interrelated by $D_{a}\left(t_{0}\right) \subseteq D_{s}\left(t_{0}\right)$ and $D\left(t_{0}\right)=D_{a}\left(t_{0}\right)$, for all $t_{0} \in R_{\text {. }}$.

(b) If the state $x=0$ of the system (1) possessing the Weak Smoothness Property is uniformly asymptotically stable and its domain $D_{a}\left(R_{i}\right)$ of uniform asymptotic stability on $R_{i}$ satisfies $D_{a}\left(R_{i}\right) \subseteq S\left(R_{i}\right)$ then its domains $D_{a}\left(R_{i}\right), D_{s}\left(R_{i}\right)$ and $D\left(R_{i}\right)$ are interrelated by $D_{a}\left(R_{i}\right) \subseteq D_{s}\left(R_{i}\right)$ and $D\left(R_{i}\right)=D_{a}\left(R_{i}\right)$.

PROOF. Let the system (3.1) possess the Weak Smoothness Property. Let $x=0$ be asymptotically stable and $D_{a}\left(t_{0}\right) \subseteq S\left(t_{0}\right)$ for all $t_{0} \in R_{i}$. Let $\left(t_{0}, \mathrm{x}_{0}\right) \in R_{i} \times D_{a}\left(t_{0}\right)$ be arbitrary. Continuity of $\mathbf{x}\left(t ; t_{0}, \mathrm{x}_{0}\right)$ in $\left(t_{0}, \mathrm{x}_{0}\right) \in R_{2} \times S\left(t_{0}\right), \quad D_{a}\left(t_{0}\right) \subseteq S\left(\mathrm{t}_{0}\right)$ and $\mathrm{x}_{0} \in D_{a}\left(t_{0}\right)$ imply $\max \left[\left\|\mathbf{x}\left(t ; t_{0}, x_{0}\right)\right\|: t \in R_{0}\right]<\infty$. Let $\epsilon=2 \max \left[\left\|\mathbf{x}\left(t ; t_{0}, x_{0}\right)\right\|: t \in R_{0}\right]$. Hence, $x_{0} \in D_{s}\left(t_{0}, \epsilon\right)$ due to (a-1) of Definition 2, which implies $x_{0} \in D_{s}\left(t_{0}\right)$ in view of (a-3) of Definition 2. Altogether, $\mathrm{x}_{0} \in D_{a}\left(t_{0}\right)$ yields $\mathrm{x}_{0} \in D_{s}\left(t_{0}\right)$ that proves $D_{a}\left(t_{0}\right) \subseteq D_{s}\left(t_{0}\right)$ for all $t_{0} \in R_{i}$. This result and (a) of Definition 3 complete the proof of the statement under (a).

Let $\mathrm{x}=0$ be uniformly asymptotically stable on $R_{i}$ and $D_{a}\left(R_{i}\right) \subseteq S\left(R_{i}\right)$. Let $\mathrm{x}_{0} \in D_{a}\left(R_{i}\right)$ be arbitrary. Hence, $\max \left[\left\|\mathbf{x}\left(t ; t_{0}, \mathrm{x}_{0}\right)\right\|:\left(t, t_{0}\right) \in R_{0} \times R_{i}\right]<\infty$ due to continuity of $\mathbf{x}\left(t ; t_{0}, \mathbf{x}_{0}\right)$ in $\left(t, t_{0}\right) \in R_{0} \times R_{i}$ and $\mathrm{x}_{0} \in D_{a}\left(R_{1}\right)$. Let $\epsilon=2 \max \left[\left\|\mathbf{x}\left(t ; t_{0}, \mathrm{x}_{0}\right)\right\|:\left(t, t_{0}\right) \in R_{0} \times R_{i}\right] \in R^{+}$so that obviously $\mathrm{x}_{0} \in D_{s}\left(\epsilon, R_{l}\right)=\cap\left[D_{s}\left(t_{0}, \epsilon\right): t_{0} \in R_{l}\right]$ and therefore $\mathrm{x}_{0} \in D_{s}\left(R_{i}\right)$ (Definition 3). The result that $\mathrm{x}_{0} \in D_{a}\left(R_{i}\right)$ implies $\mathrm{x}_{0} \in D_{s}\left(R_{i}\right)$ proves $D_{a}\left(R_{i}\right) \subseteq D_{s}\left(R_{i}\right)$ and $D\left(R_{\mathrm{i}}\right)=D_{a}\left(R_{i}\right)$ (due to Definition 3). This completes the proof. 


\section{REFERENCES}

[1]GRUIC', L., Novel development of Lyapunov stability of motion, Int. Journal of Control, 22, 4 (1975), 525-549.

[2] GRUIC, L. T., On absolute stability and the Aizerman conjecture, Automatica, 17, 2 (1981), 335349.

[3] GRUJC', L. T., Concepts of stability domains (in Serbo-Croatian), Automatika, Zagreb, 26, 1-2 (1985), 5-10.

[4] GRUJC, L. T., Solutions to Lyapunov stability problems: Nonlinear systems with globally differentiable motions, in The Lyapunov Functions Method and Applications, Eds. P. Borne and V. Matrosov, J. C. Baltzer, AG, Scientific Publishing Co., IMACS (1990), 19-27.

[5] GRUJC', L. T., Solutions to Lyapunov stability problems: Nonlinear systems with differentiable motions, in Proc. 13th IMACS World Congress on Computation and Applied Mathematics, 3 (1991), 1228-1231.

[6] GRUJC', L. T., The necessary and sufficient conditions for the exact construction of a Lyapunov function and the asymptotic stability domain, Proc. 30th IEEE Conference on Decision and Control, 3 (1991), 2885-2888.

[7] GRUJĆ, L. T., On solutions of Lyapunov stability problems, Facta Universitatis, Ser.: Mechanics, Automatic Control and Robotics, University of Niš, Niš, Serbia, 1, 2 (1992), 121-138.

[8] GRUJíC, L. T., Exact determination of a Lyapunov function and the asymptotic stability domain, Int. J. Systems Science, 23, 11 (1992), 1874-1888.

[9] GRUIC, L. T., Exact both construction of Lyapunov function and asymptotic stability domain determination, Proc. 1993 IEEE International Conference on Systems, Man and Cybernetics, 1 (1993), 331-336.

[10]GRUJC, L. T., Solutions to Lyapunov stability problems: nonlinear systems with continuous motions, Int. J. of Mathematics and Mathematical Sciences, 17, 3 (1994), 587-596.

[11]GRUJC, L. T., Solutions to Lyapunov stability problems of sets: nonlinear systems with differentiable motions, Int. J. of Mathematics and Mathematical Sciences, 17, 1 (1994), 103-112.

[12] GRUJC, L. T., MARTYNYUK, A. A. and RIBBENS-PAVELLA, M., Large-Scale Systems Stability under Structural and Singular Perturbations (in Russian), Naukova dumka, Kiev, 1984 (English translation: Springer Verlag, New York, 1987).

[13] GRUJĆ, L. T. and MICHEL, A. N., Exponential stability and trajectory bounds of neural networks under structural variations, IEEE Transactions on Circuits and Systems, 38, 10 (1991), 1182-1192.

[14] GRUIĆ, L. T. and RIBBENS-PAVELLA, M., Asymptotic stability of large-scale systems with application to power systems: Part 1; domain estimation, Eleo. Power and Energy Systems, 1, 3 (1979), 151-157.

[15]HAHN, W., Stability of Motion, Springer-Verlag, Berlin, 1967.

[16]LAKSHMIKANTHAM, V. and LEELA, S., Differential and Integral Inequalities, Academic Press, New York, 1969.

[17]LaSALLE, J. P. and LEFSCHETZ, S., Stability by Liapunov's Direct Method, Academic Press, New York, 1961.

[18]MIILER, R. K. and MICHEL, A. N., Ordinary Differential Equations, Academic Press, New York, 1982.

[19] VANELLI, A. and VIDYASAGAR, M., Maximal Lyapunov functions and domains of attraction for autonomous nonlinear systems, Automatica, 21, 1 (1985), 69-80.

[20]ZUBOV, V. I., Methods of A. M. Liapunov and Their Applications, P. Noordhoff Ltd., Groningen, 1964. 


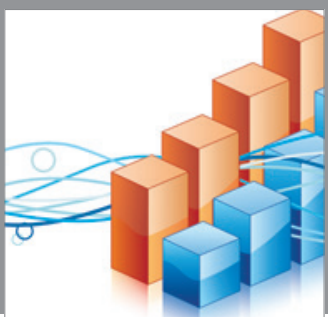

Advances in

Operations Research

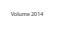

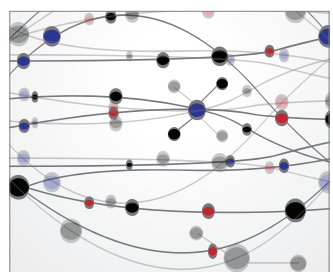

\section{The Scientific} World Journal
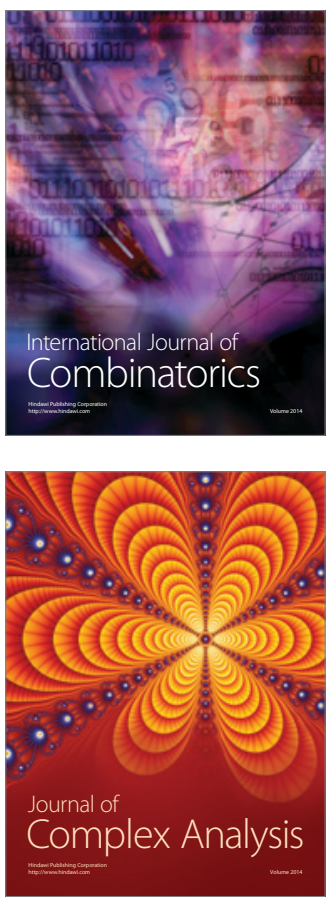

International Journal of

Mathematics and

Mathematical

Sciences
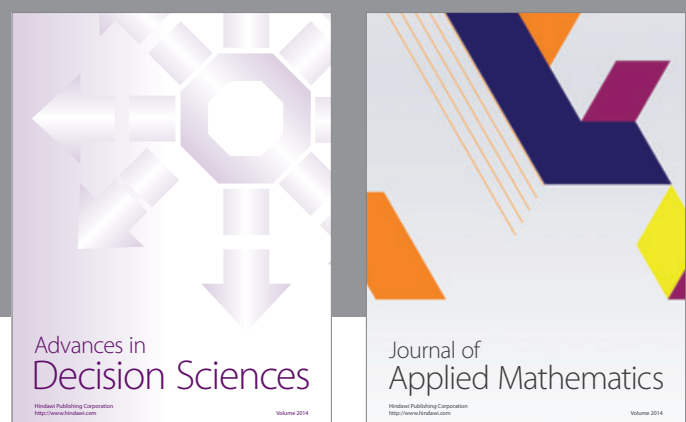

Journal of

Applied Mathematics
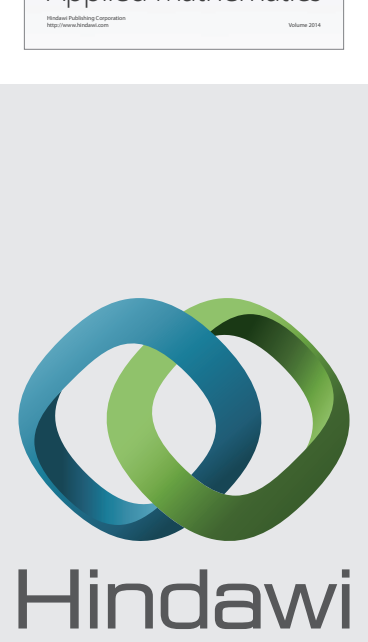

Submit your manuscripts at http://www.hindawi.com
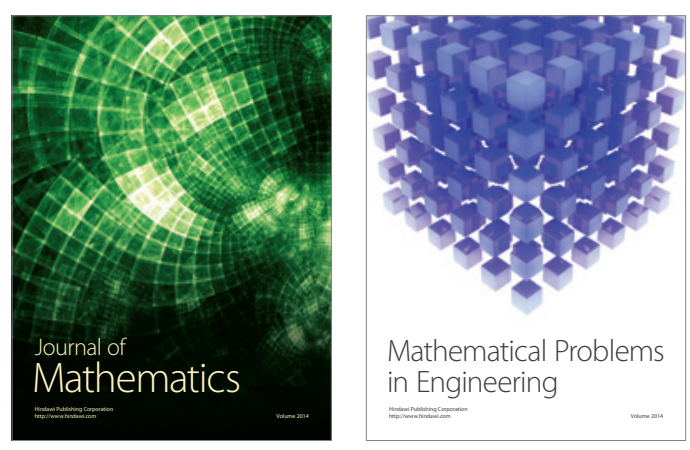

Mathematical Problems in Engineering
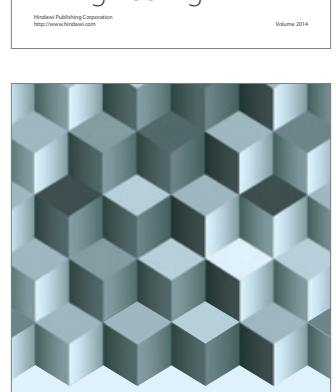

Journal of

Function Spaces
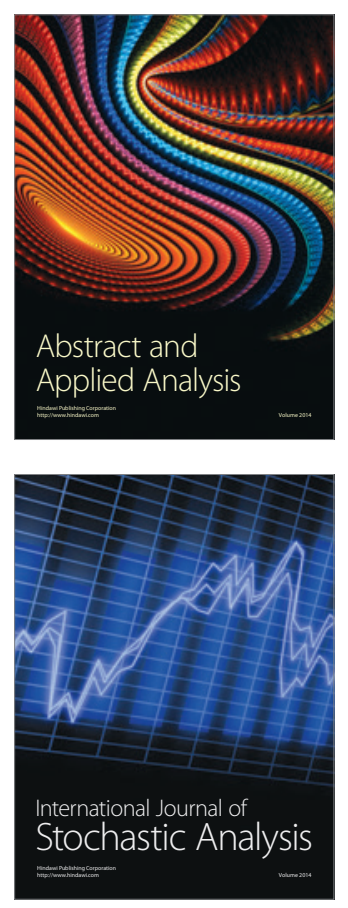

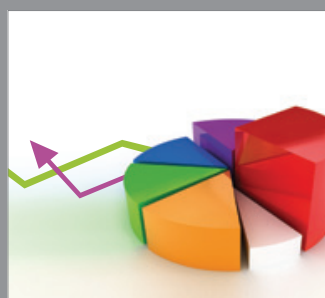

ournal of

Probability and Statistics

Promensencen
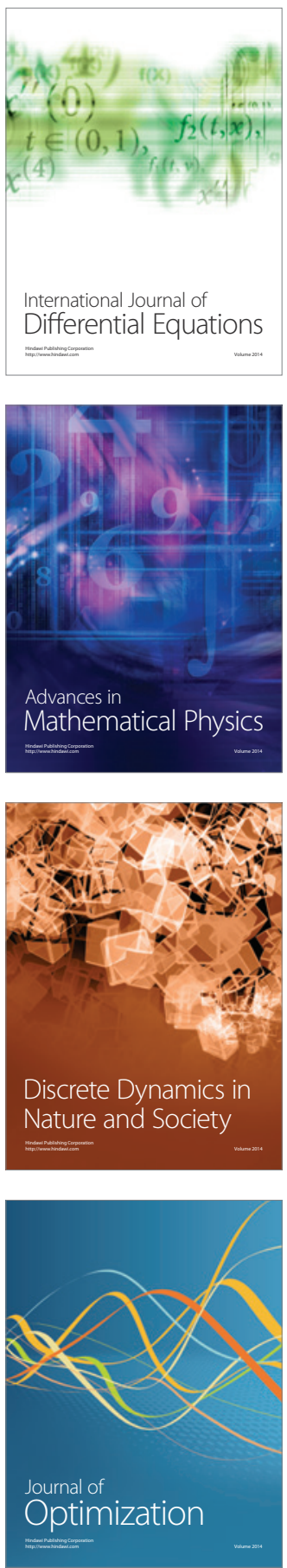